\title{
Purkinje Cell Activity During Suppression of Voluntary Eye Movements in Rhesus
}

\section{Macaques}

Eric Avila ${ }^{1,2 \#}$, Nico A. Flierman ${ }^{1,2 \#}$, Peter J. Holland ${ }^{3}$, Pieter R. Roelfsema ${ }^{1,4,5}$, Maarten A.

Frens $^{2}$, Aleksandra Badura ${ }^{1,2^{*}}$ and Chris I. De Zeeuw ${ }^{1,2^{*}}$

${ }^{1}$ Netherlands Institute for Neuroscience, Amsterdam, The Netherlands

${ }^{2}$ Department of Neuroscience, Erasmus MC, Rotterdam, The Netherlands

${ }^{3}$ School of Psychology, University of Birmingham, Birmingham, UK

${ }^{4}$ Department of Integrative Neurophysiology, VU University, Amsterdam, The Netherlands

${ }^{5}$ Department of Psychiatry, Academic Medical Centre, Amsterdam, The Netherlands

\# These authors contributed equally to this work.

EA current address: Center for Neural Science, New York University, New York, USA.

*Correspondence: Aleksandra Badura (a.badura@erasmusmc.nl)

Department of Neuroscience, Erasmus MC

Wytemaweg 80, $3015 \mathrm{CN}$ Rotterdam

tel: 0031-(0)10-7043309

Chris I De Zeeuw (c.de.zeeuw@nin.knaw.nl)

Netherlands Institute for Neuroscience

Meibergdreef 47,1105 BA, Amsterdam

tel: 0031-(0)20- 5665500 


\begin{abstract}
Conscious control of actions helps us to reach our goals by suppressing responses to distracting external stimuli. The cerebellum has been suggested to complement cerebral control of inhibition of targeted movements (conscious control), though by what means, remains unclear. By measuring Purkinje cell (PC) responses during antisaccades, we show that the cerebellum not only plays a role in the execution of eye movements, but also in during the volitional inhibition thereof. We found that simple spike (SS) modulation during instruction and execution of prosaccades and antisaccades was prominent in PCs of both medial and lateral cerebellum, showing distinct, timeordered sequences, but each with different sensitivities for execution and trial-history. SS activity in both regions modulated bidirectionally, with both facilitation (increasing SS firing) and suppression (decreasing SS firing) PCs showing firing-rate changes associated with instruction and execution, respectively. These findings show that different cerebellar regions can contribute to behavioral control and inhibition, but with different propensities, enriching the cerebellar machinery in executive control.
\end{abstract}

Keywords: Cerebellum, flexible behavior, executive control, antisaccade, monkeys. 


\section{Introduction}

In a dynamic environment, volitional control of behavior is necessary to make flexible, welladapted choices. In order to accomplish internal goals, voluntary behavior often requires suppression of reflexive responses to external stimuli, a hallmark of conscious, executive control ${ }^{1}$. For example, when we focus our visual attention in everyday life, we suppress our natural tendency to make a saccade to nearby moving or static objects in order to keep our gaze fixed on the target of choice ${ }^{2}$. In a laboratory setting, we can measure this complex behavior using the antisaccade task. It requires participants to refrain from looking at a suddenly appearing target ('reflexive response') and instead execute a saccade to the (unmarked) mirror position of that target ('internal goal' $)^{2-4}$. This ability to voluntarily inhibit the urge to look at the target and instead make a saccade away from it is part of executive control function and supports goal-directed behavior. It is disrupted in patients suffering from neurological and/or psychiatric disorders, such as schizophrenia or attention-deficit hyperactivity disorder, that are characterized by difficulties in response inhibition ${ }^{5-8}$.

Similar to that of other volitional movements, antisaccade control is regarded to be organized top-down in that the cerebral cortex has been claimed to initiate such movements ${ }^{9,10}$. This notion is supported by observations that antisaccade related activity has been found in various parts of the cortex, including dorsolateral prefrontal cortex (DLPFC), frontal eye fields (FEFs), and supplementary eye fields $(\mathrm{SEFs})^{6,9-14}$. However, given that all these regions are both downstream and upstream connected with the cerebellum ${ }^{15}$, the cerebellum may well form an additional hub in this voluntary motor control circuitry ${ }^{16-20}$. This possibility is corroborated by recent findings that the cerebellum does not only participate in movement execution, but also in 
movement planning ${ }^{21-25}$. Yet, whether and how the cerebellum may contribute to the inhibition of a prepotent response and executive control thereof remains to be elucidated. Moreover, to what extent different parts of the cerebellum might contribute differentially to the planning and execution of such complex movements is also unclear ${ }^{21,26-31}$. Some studies indicate that executions of simple or reflexive movements may be controlled by Purkinje cells (PCs) in the medial part of the cerebellum, whereas complex behaviors, which require instruction, inhibition and planning (executive control), might be controlled by more lateral parts ${ }^{30-34}$. Others however, advocate that both the simple and complex movements can be controlled by the same group of Purkinje cells ${ }^{21,26}$, the location of which in the cerebellar cortex is determined by the PC-to-effector pathway ${ }^{35}$. Settling these questions would require simultaneous monitoring of spiking activity of $\mathrm{PCs}^{36}$ in both medial and lateral cerebellar areas during a motor task that includes both the execution of a simple reflex and a more complex form of inhibitory planning of movements related to the same effector.

Here, we investigated the hypotheses that (1) PCs in the cerebellum do not only play a role in the execution of reflexive eye movement responses, but also during the volitional inhibition of such stimulus - response eye movements; and (2) that PCs in medial and lateral cerebellum differentially contribute to these two tasks. More specifically, we set out to study PC modulation in the medial (oculomotor vermis or OMV) and lateral (crus-I/II) cerebellum of non-human primates (macaca mulatta) during the generation of both prosaccades and antisaccades (Fig. 1). The animals were trained to make either a prosaccade or an antisaccade to a mirror 'invisible' target depending on an initial color cue. During the entire paradigm we recorded both simple spike (SS) and complex spike (CS) activity of PCs, which are mediated by the mossy fiber and climbing fiber pathway, respectively ${ }^{36}$. 
We found that SS modulation during the instruction and the execution periods of prosaccade and antisaccade eye movements was prominent in PCs of both medial and lateral cerebellum. SS activity in both regions modulated in both a facilitating and suppressing manner, a feature that is common in sensorimotor tasks that require bidirectional control in the PC-to-effector pathway $^{37-42}$. In both regions, the PCs of the facilitation category showed a prominent increase in firing rate just before target appearance during the instruction period. In addition, in both regions SS activity displayed distinct, time-ordered, sequences that were stable across many trials, which can be considered a recurring motif of population activity when keeping track of timing is critical $^{43-45}$. However, various other parameters of the SS activity modulations differed between medial and lateral cerebellum. PCs in the lateral cerebellum showed a greater firing rate during the execution of saccades. In addition, the same cells in the lateral cerebellum displayed modulated SS signals in the instruction period in both pro- and antisaccade trials and showed more prominent saccade-related activity at the population level. PCs in the medial cerebellum were more sensitive to trial history, and PCs of the suppression category in this region showed a relatively late SS modulation during execution of both pro- and antisaccades, in line with previous findings ${ }^{25,41,42}$. CS activity modulated during pro- and antisaccade trials in both cerebellar regions throughout the task, but it showed a higher level of reciprocity with respect to SS activity in the lateral cerebellum. Our results indicate that PCs in the OMV of the medial cerebellum and those in crus-I/II of the lateral cerebellum differentially contribute to the instruction and execution of reflexive behavior and the suppression thereof. These findings highlight that different cerebellar regions can contribute to both simple and complex forms of related behaviors, yet with different propensities. Moreover, they are adding to the growing evidence of the role of the cerebellum in executive 

available under aCC-BY-NC-ND 4.0 International license.

control, and they are challenging us to explore cerebellar activity under conditions where response inhibition is hampered. 


\section{Results}

\section{Behavior during pro- and antisaccades}

We trained two adult, male rhesus macaques (referred to as monkeys Mo and $\mathrm{Mi}$ ) to perform a randomized, interleaved pro- and antisaccade task. Correct behavior required either the execution of a saccade to a single visual target in one of 8 directions (prosaccade) or the suppression of the prepotent saccade to a visible target and redirection of gaze to an unmarked mirror position (antisaccade, Fig. 1a). A trial started with the appearance of a central fixation point and the trial condition was indicated by the fixation point color (red for prosaccade, presented in figures in magenta; green for antisaccade, presented in figures in blue; colors were adjusted for presentation to accommodate color-blind readers). After this instruction period (300-500 ms), a target would appear randomly at 1 of 8 different locations separated $45^{\circ}$ from each other, while monkeys maintained fixation for $100 \mathrm{~ms}$ more before the central fixation point turned gray (referred to as motor planning period), serving as the "go-cue". Animals had $500 \mathrm{~ms}$ to execute the pro- or antisaccade (execution or saccade period) before the trial was aborted. This task design temporally separates the initial neural representation of the stimulus from the subsequent motor preparation and execution ${ }^{46}$.

We removed trials with saccades with reaction times of less than $100 \mathrm{~ms}$ to avoid contamination from anticipatory saccades; these reflect a visual, involuntary reflex towards a novel stimulus in the environment and do not represent correctly planned saccades, during which the monkeys delay their eye movements until the presentation of the "go-cue" 47,48 . We also removed error trials, as we did not have enough power to analyze them due to the accuracy across the experiments $(94.1 \pm 5.6 \%$ and $94.4 \pm 5.3 \%$ for $\mathrm{Mo}$ and $\mathrm{Mi}$, respectively). The eye movements 


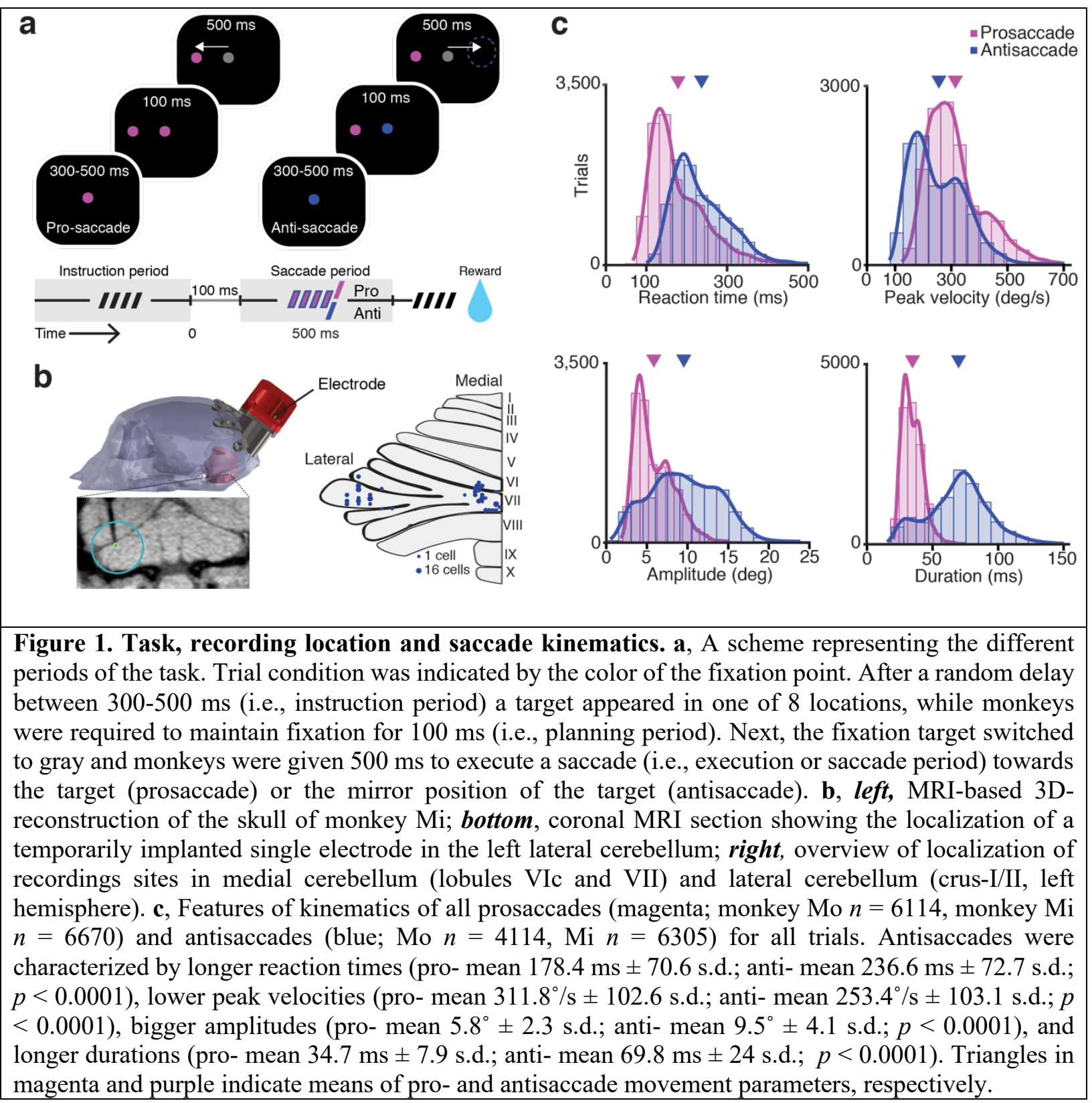


Purkinje cells in both medial and lateral cerebellum modulate their activity during execution of pro- and antisaccades, but with different characteristics

While the monkeys performed the task, we recorded from 90 PCs in medial and 72 PCs in lateral (Crus-I/II) cerebellum during 122 sessions (Fig. 1b); recording sessions started when the animals reached a performance of at least $80 \%$ after the initial weeks of training. A neuron was considered to be task-related if SS activity during the beginning of the saccade period (i.e., from saccade onset to $150 \mathrm{~ms}$ after saccade onset) was significantly different from the intertrial interval period (see Methods). Approximately half of the cells recorded in both areas fulfilled this criterion $[\mathrm{n}=40$ (43\%) for medial cerebellum and $n=38(45 \%)$ for lateral cerebellum]. All subsequent analyses were performed only for these cells unless noted otherwise $(n=78$ PCs).

Many PCs showed SS modulations in relation to peak velocity, amplitude and duration of pro- and/or antisaccades, but on average no significant differences were observed between medial and lateral cerebellum in this respect or preference for directional preference (see also below). We regressed the firing rate in the two areas for either amplitude or peak velocity and compared the corrected and uncorrected firing rate using the regression coefficient ( $($ ) for pro- and antisaccade in both areas (corrected firing $=$ firing rate/regression coefficient). We found no correlations between firing rate and either saccade amplitude or saccade peak velocity in the two areas between the two conditions. This allowed us to directly compare them by pooling the mean firing rate for each cell during pro- and antisaccades (Supplementary Figure 1a-d and Supplementary Table 1). Activity of PCs obtained during the first $150 \mathrm{~ms}$ after saccade onset, was significantly higher in the lateral cerebellum in both conditions (mean and s.d., medial pro $66.7 \pm 30 \mathrm{spks} / \mathrm{s}$, lateral pro $69.3 \pm 39 \mathrm{spks} / \mathrm{s}, p=0.04$; medial anti $68 \pm 36 \mathrm{spks} / \mathrm{s}$, lateral anti $71.8 \pm 39 \mathrm{spks} / \mathrm{s}, p=0.03$ for pro- and antisaccades, Wilcoxon rank sum test). 
We classified the PCs into two different groups depending on whether they decreased (suppression PCs) or increased (facilitation PCs) their firing rate after saccade onset (Figure 2). The activity profile of the PCs was the same during pro- and antisaccades in both areas except for two PCs in the lateral cerebellum, which showed a mixed firing behavior; these two cells were included in their respective category (facilitation or suppression) for further analyses. The mean latencies of SS activity of suppression PCs within medial and lateral cerebellum during prosaccades, defined as the largest trough after saccade onset, were similar to those during antisaccades (mean \pm s.d.: Medial pro $87 \pm 70 \mathrm{~ms}$ vs Medial anti $93 \pm 67 \mathrm{~ms}, p=0.83$, Wilcoxon Rank sum test; Lateral pro $139 \pm 40 \mathrm{~ms}$ vs Lateral anti $127 \pm 42 \mathrm{~s}, p=0.1$, Wilcoxon Rank sum test; Supplementary Figure 2). However, when we compared the two regions, we found that the latencies of the suppression PCs in the lateral cerebellum (mean \pm s.d.: $133 \pm 40 \mathrm{~ms}$ ) were significantly longer than those in the medial cerebellum ( $89 \pm 68 \mathrm{~ms} ; p=0.01$, Wilcoxon rank sum). In contrast, facilitation PCs peaked their activity after saccade onset on average at similar times during pro- and antisaccades and similarly in both medial and lateral cerebellum (medial vs lateral for pro- and antisaccade cells $p=0.2$ and $p=0.9$, respectively, Wilcoxon Rank sum test; Fig. 2e-f); all occurring approximately $100 \mathrm{~ms}$ after saccade onset (mean \pm s.d.: Medial pro: 105 $\pm 41 \mathrm{~ms}$ vs Lateral pro $84 \pm 48 \mathrm{~ms}$; Medial anti: $104 \pm 33 \mathrm{~ms}$ vs Lateral anti $106 \pm 43 \mathrm{~ms}$ ). 


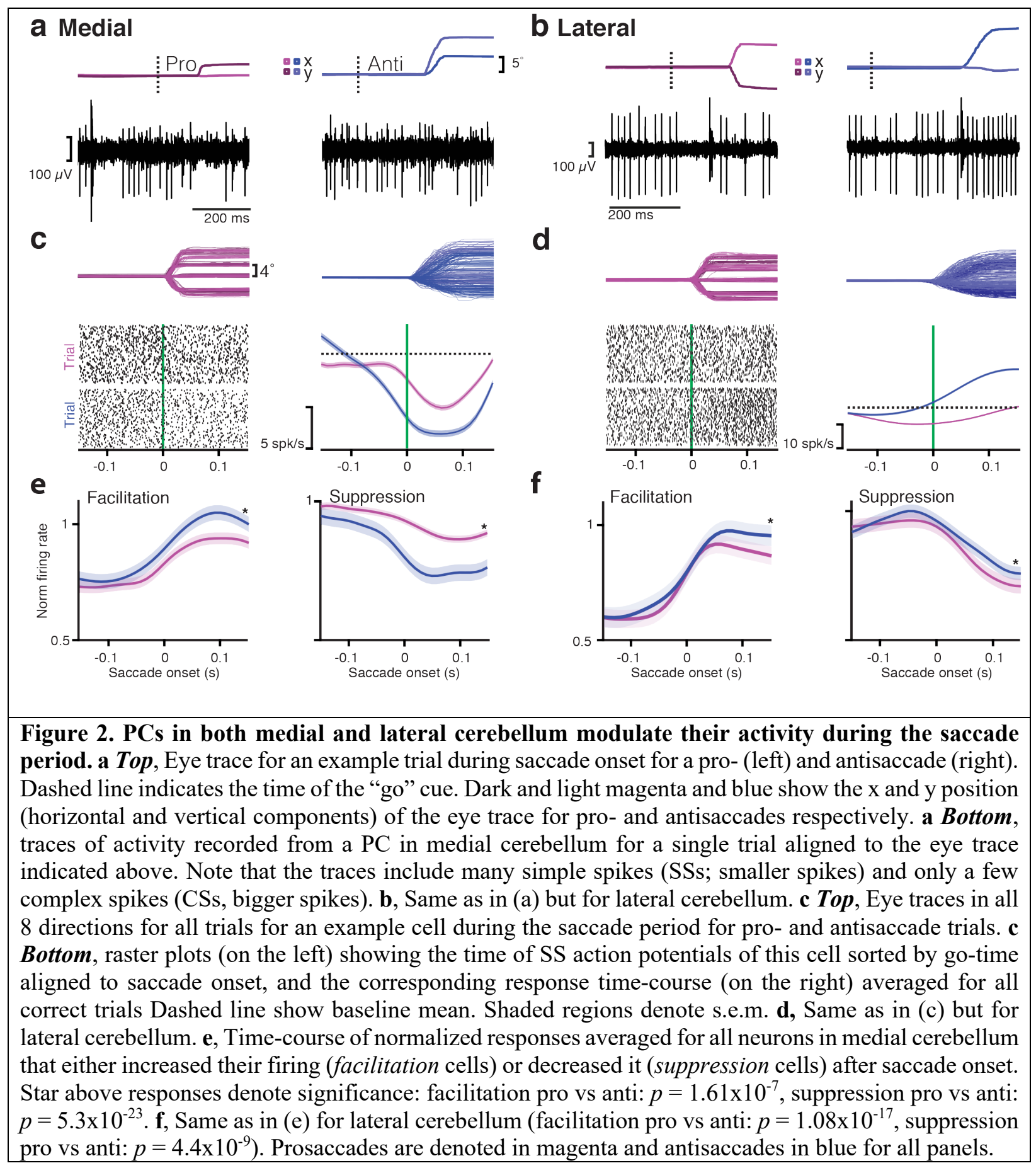


Purkinje cells in both medial and lateral cerebellum modulate their activity during instruction of pro- and antisaccades, but with different characteristics

We next assessed SS activity during the instruction period for the PCs that modulated their activity up or down during the saccade period (for details, see Methods). Given the variable duration of the instruction period, which varied between 300 and $500 \mathrm{~ms}$, we analyzed the last 300 ms of this epoch, before the onset of the target, for all cells (Fig. 1a). Here, we also encountered diverse neural responses in both regions and classified the activity of these neurons in the same way as for the saccade period depending on whether they increased (facilitation cells) or decreased (suppression cells) their firing after instruction onset compared to the activity during the intertrial interval. In the medial cerebellum, the percentages of PCs that responded with increases (10\%) or decreases (11\%) of their SS activity during instruction of prosaccades were similar to those during the instruction of antisaccades (11\% and 7\%, respectively, Fig. 3a, Supplementary Table 2). The same was true for lateral cerebellum, where the percentages of facilitation and suppression PCs were similar in the prosaccade and antisaccade trials $(17 \%$ and $14 \%$ prosaccade; $18 \%$ and $15 \%$ antisaccade, facilitation and suppression respectively, Supplementary Table 2). Overall, we found more significantly modulated PCs during the instruction period in the lateral cerebellum than in the medial cerebellum (Medial vs Lateral, $p=0.03$; Mann-Whitney test), suggesting a more prominent role for PC modulation in the lateral cerebellum during instruction. This is congruent with the finding that in the lateral cerebellum, the levels of PCs modulation during the execution period, of both types of trials, are higher during the instruction period (Fig. 3b-c). Accordingly, we found for both prosaccades and antisaccades in the lateral cerebellum, but only for prosaccades in the medial cerebellum, that the maximum firing rate of the neurons was significantly lower during the instruction period than the execution period (Medial pro $p=0.03$, anti $p=0.07$, Lateral 
pro $p=0.002$, anti $p=0.01$, Wilcoxon rank sum test) (Fig. 3c). In addition to the differences between PC activities in the medial and lateral cerebellum associated with the instruction period highlighted above, there were also several interesting differences that were not related to the region involved. For example, SS modulation during the instruction period in both cerebellar areas was more prominent during the instruction for antisaccades than that for prosaccades. The changes in SS firing rate were significantly greater for the antisaccade trials as compared to those for the prosaccade trials in both areas (medial: facilitation $p=4.4 \times 10^{-04}$, suppression $p=1.03 \times 10^{-16}$; lateral: facilitation $p=0.01$, suppression $p=0.04$; K-S test, Fig. 3a). Unlike the execution period, which was dominated by suppression cells, during instruction the contribution of the facilitation cells to preparation of the antisaccades appeared most prominent. Indeed, when we subtracted the prosaccade SS modulation from the SS modulation during antisaccades (antisaccade SS activityprosaccade SS activity), facilitation PCs of both medial and lateral cerebellum showed a prominent change in firing rate just before target appearance during the instruction period (Fig. 3b) and these changes were significantly less pronounced for suppression cells (facilitation PCs versus suppression PCs in medial cerebellum $p=4.4 \times 10-6$; facilitation PCs versus suppression PCs in lateral cerebellum $p=0.004$; Wilcoxon Rank sum test).

SS activity modulation bridged the interval between the instruction and the saccade epoch through ramping (Fig. 3d). We quantified the ramping of SS activity by fitting a linear model to the firing rate during the last $300 \mathrm{~ms}$ of the instruction period, cells were classified as having bridging when $\mathrm{R}^{2}$ was higher than 0.75 , we found that bridging modulation was quite rare in the medial cerebellum and more prevalent in the lateral cerebellum (Fig. 3e); this held true for both prosaccade and antisaccade trials (both comparisons $p=0.02 ; \chi^{2}$ test, medial: $n$ (pro) $=3, n$ (anti) $=2$; lateral: $n($ pro $)=11, n($ anti $)=10)$. 


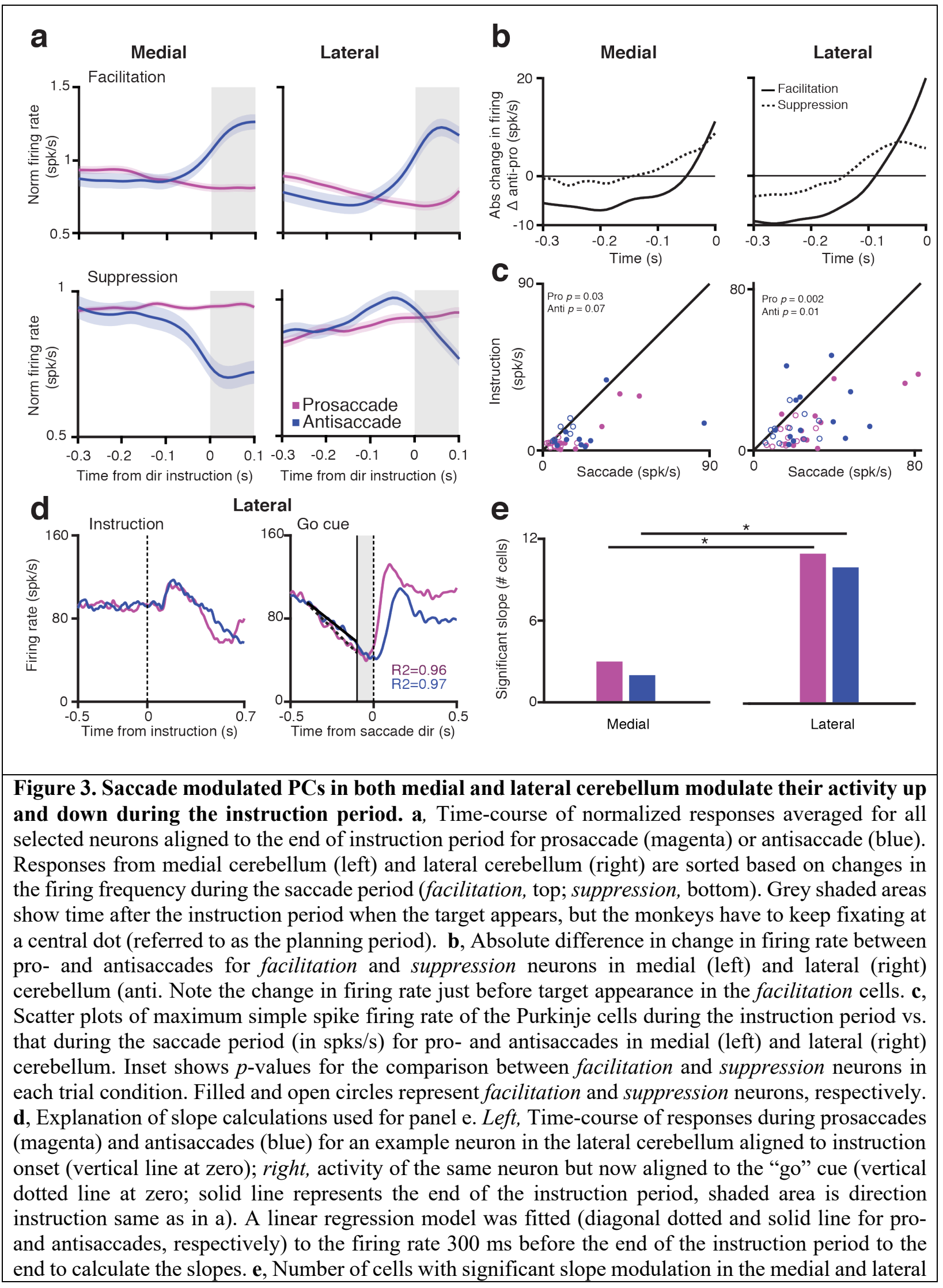


cerebellum ( $\chi^{2}$ test for proportions) are higher in the lateral cerebellum for both prosaccade (magenta) and antisaccade (blue) trials ( $p=0.02$ for both comparisons).

We conclude that PCs in both medial and lateral cerebellum modulate their activity during instruction of pro- and antisaccades, with a relatively prominent role for the facilitation cells during the instruction of antisaccades. Yet, PCs in the lateral cerebellum differ from those in the medial cerebellum in that they have a relatively stronger modulation in both the instruction and execution periods.

Population of Purkinje cells in the lateral, but not in medial, cerebellum denotes trial identity based on simple spike activity

Given the response profiles described above we wondered to what extent the trial conditions were represented by the populations of PCs in the medial and lateral cerebellum. We compared the differences in neural activity between pro- and antisaccades in both areas by computing a modulation ratio between the responses during the saccade period in the two types of trials, defined as the ratio between the response during pro- and antisaccades (Fig. 4a, Methods). Values of 1 mean that both responses are the same, values higher than 1 mean that prosaccade activity was higher, and below 1 that antisaccade activity was higher. Medial PCs had somewhat different responses when comparing pro- and antisaccade activity in contrast to lateral PCs (mean of facilitation PCs in medial cerebellum $0.91 \pm 0.1$ s.d. versus that in lateral cerebellum $0.88 \pm 0.3$, $p=0.03$; mean of suppression PCs in medial cerebellum $1.16 \pm 0.2$ versus that in lateral cerebellum $1 \pm 0.2, p=8.02 \times 10^{-04}$; one-tailed K-S test, selected neurons Supplementary Table 2). 
To further quantify the differences between the medial and lateral cerebellum and address their potential meaning, we computed a binomial proportion test as a function of time between pro- and antisaccades for the same neurons to better capture the time at which the spiking was significantly different and show if, and how well, single PCs discriminate between both conditions in both regions. The responses of single PCs differ strikingly between the two areas and the two different activity populations, especially for the PCs that showed significantly different activity between pro- and antisaccades (Fig. 4b). This is for neurons that changed their firing during the saccade execution period and had a firing rate significantly different between the two conditions. Unlike suppression PCs, facilitation PCs in the lateral, but not in the medial, cerebellum exhibited prominent contrasting activity mainly present around saccade onset (Fig. 4b-c). Apparently, at the population level, the activity of medial cerebellum could not discriminate between pro- and antisaccade trials, even though individual PCs in that region showed significant differences between the conditions (Fig. 4b). In contrast, the population of PCs in the lateral cerebellum was able to effectively discriminate between the two types of trials during saccade execution only, reaching peak separation at $70 \mathrm{~ms}$ after saccade onset (Fig. 4c). This is quite surprising, because both types of PCs (i.e., facilitation and suppression cells) in both areas exhibit saccade-related activity, but only the facilitation cells in the lateral parts of the cerebellum contain information about the trial at the population level. 

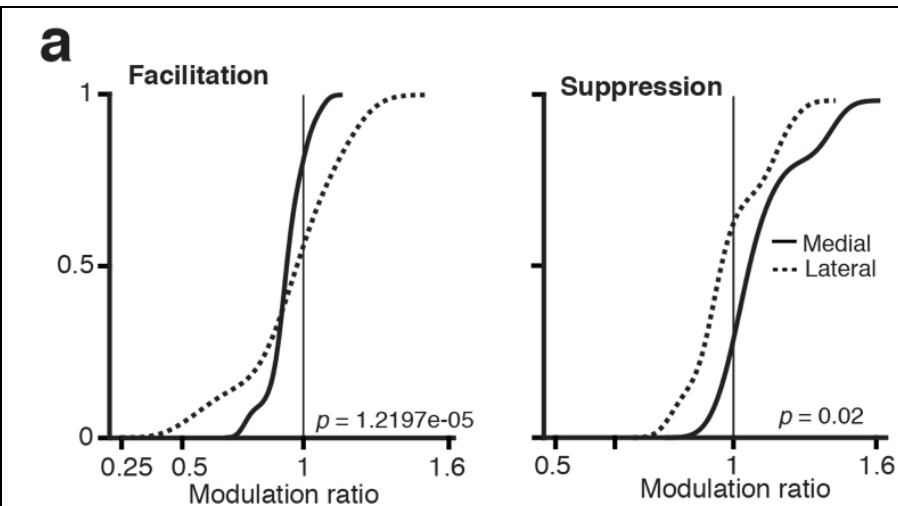

b

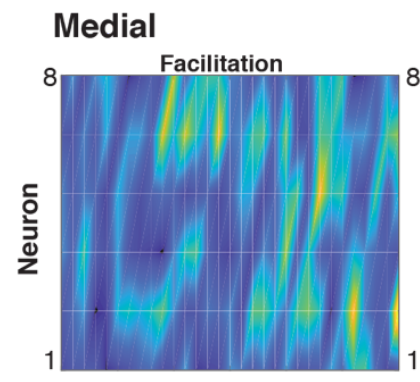

Lateral
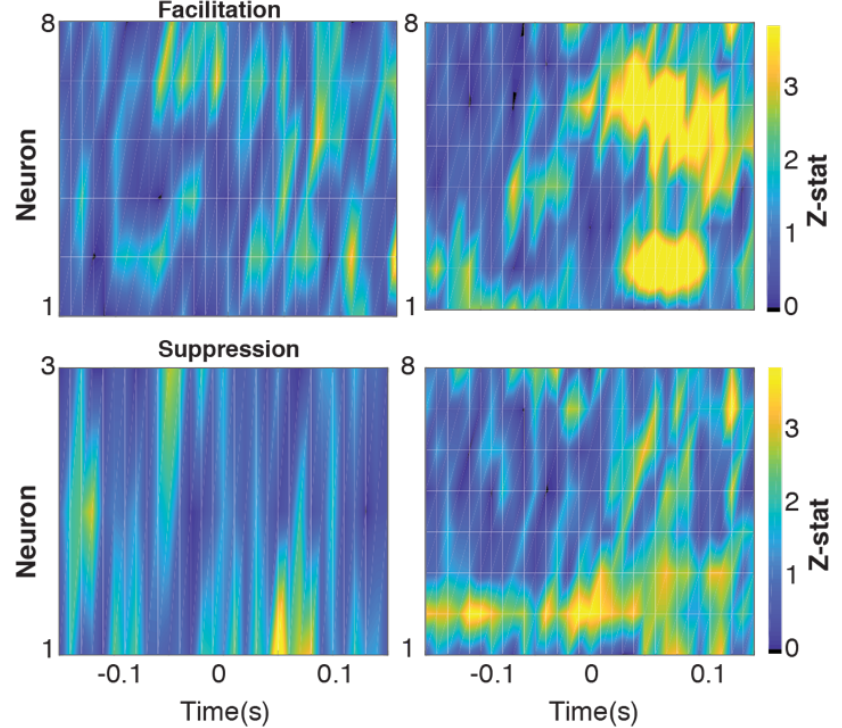

C

Medial
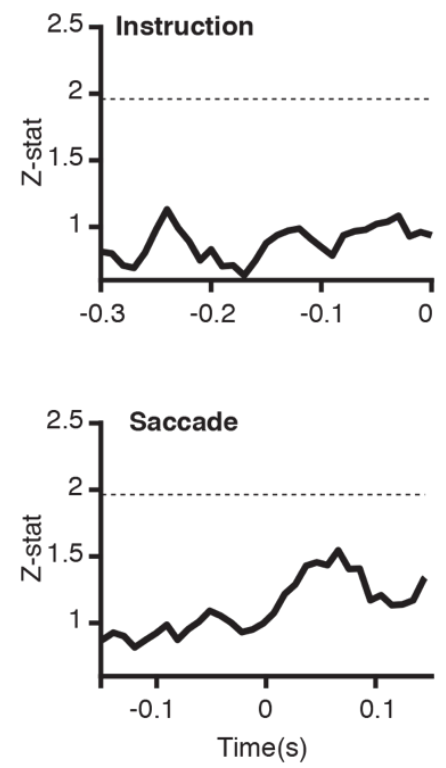

Figure 4. Population encoding of SS modulations in lateral versus medial cerebellum. a, Cumulative distribution of the SS modulation ratio for medial and lateral cerebellum for facilitation and suppressive PCs. Insets show $p$-values for the comparison between medial and lateral cerebellum (two sample K-S test). b, Z-statistic of a binomial probability distribution comparison between PCs that exhibited significant difference in activity between pro- and antisaccades in medial (left) and lateral (right) cerebellum separate for facilitation and suppressive responses (top and bottom respectively). Color bar is set at twice the significance value (1.96). c, Z-statistic of a binomial proportion test during the saccade period as a function of time for pro- and antisaccades for medial (left) and lateral (right) cerebellum during the instruction (top) and saccade (bottom) period for significantly different neurons (Methods). 
Purkinje cells in medial cerebellum are sensitive to trial history

It has been suggested that the PC response on a given trial may be modulated by the recent history of different trial types ${ }^{42}$. We showed above that the population response in lateral cerebellum areas is different for pro- and antisaccades, we wanted to know if the PC modulation was sensitive to trial history, given that the monkeys exhibit a low error performance. We performed trial-by-trial analysis of the SS firing rate of all PCs, comparing the absolute difference in SS spiking activity between trial $n$ and one trial back (n-1), and trial $n$ and two trials back $(n-2)$. We included in our analysis one or two trials back to assess if there is an influence of previous trial(s) on the current one, especially when the last trial(s) switched from prosaccade to antisaccade or vice versa (current trial-1 or current trial-2). We did not observe any significant changes during the instruction period (Supplementary Figure 3a). During the execution period however, the PCs in the medial, but not in the lateral cerebellum, were indeed sensitive to trial history (Supplementary Figure 3b). The PCs of the medial cerebellum showed a significant increase in firing frequency when the identity of a trial differed from that of the previous trial (trial $n$ vs. $n-1$ $p=9.26 \times 10^{-9}$, trial $n$ vs. $n-2 p=1.76 \times 10^{-7}$, Kruskal-Wallis test). Specifically, in comparison to both one (n-1) and two trials back (n-2), trial $n$ showed the least amount of change in PC activity when a prosaccade condition was also preceded by a prosaccade trial (post-hoc Tukey-Kramer test). These results indicate that, in line with our hypothesis, PC modulation in the medial, more so than in the lateral cerebellum, depends on trial history (including the type of saccade that was executed). 
Purkinje cells in both medial and lateral cerebellum display sequential simple spike firing throughout the task

To further investigate the temporal response dynamics of the PCs in both regions during the saccade period, we sorted each PC by the time of the maximum response randomly selecting half of the trials and we plotted the normalized SS firing rate of all recorded neurons for pro- and antisaccade trials sorted by the time of their maximum response for both facilitation and suppression neurons using the other half of the trials (Fig. 5a, b, Methods). Close observation revealed a broad distribution of the peak responses of the individual PCs around saccade onset with each of them showing a preferred maximum response time. In both medial and lateral cerebellum, the timing of the occurrence of maximum firing rates were similar for pro- and antisaccade trials. We plotted the timing of these maximum responses all the neurons during pro and anti-saccades in each area and then compared for each neuron these timings between the two conditions (Fig. 5c-f). We did not observe any significant difference between medial and lateral cerebellum in this respect (i.e., facilitation cells for both pro- and antisaccades in medial cerebellum versus those for both pro- and antisaccade in the lateral cerebellum $p=0.3$ (see cells with cyan dots in Fig. 5a, b); suppression cells for both pro- and antisaccades in the medial cerebellum versus those of the lateral cerebellum $p=0.1$; facilitation cells of the medial cerebellum during prosaccade trials versus those of the lateral cerebellum under the same condition $p=0.15$; facilitation cells of the medial cerebellum during antisaccade trials versus those of the lateral cerebellum under the same condition $p=0.9$; suppression cells of the medial cerebellum during prosaccade trials versus those of the lateral cerebellum under the same condition $p=0.14$; and suppression cells of the medial cerebellum during antisaccade trials versus those of the lateral cerebellum under the same condition $p=0.51$, Fig. 5e-f). 
To test if the measured time of the responses did not represent an observation by chance, we randomly drew $50 \%$ of the trials and calculated to what extent PCs conserve their position in the sequence following a 100 time repetition (Supplementary Figure 4a and b). We computed a cosine similarity index between the patterns of SS maximum firing rates for all trials and compared it to the randomly resampled trials in four $75 \mathrm{~ms}$ time windows around saccade onset (two before and two after saccade onset; see Methods for details). Neurons with perfectly matched patterns would be assigned an index of "1", whereas no similarity between the sequences would be assigned a " 0 ". We found that the time at which each PC fires maximally is quite robust with the mean similarity index for both pro- and antisaccades being higher in the $4^{\text {th }}$ window after the saccade window in both the medial and lateral cerebellum (medial pro: $w 3=0.78 \pm 0.12$, w4 $=0.91 \pm 0.05$; medial anti: $\mathrm{w} 3=0.51 \pm 0.04, \mathrm{w} 4=0.93 \pm 0.01$; lateral pro: $\mathrm{w} 3=0.91 \pm 0.07, \mathrm{w} 4=0.93 \pm 0.05$ lateral anti: w3 $=0.74 \pm 0.01$, w $4=0.88 \pm 0.01$; see Supplementary Figure $4 \mathbf{c}$ for example neuron with maximum response at the end of the window). These results indicate that PCs in both medial and lateral cerebellum have a preference to fire at specific times during the task and that this pattern is relatively well preserved across trials. 


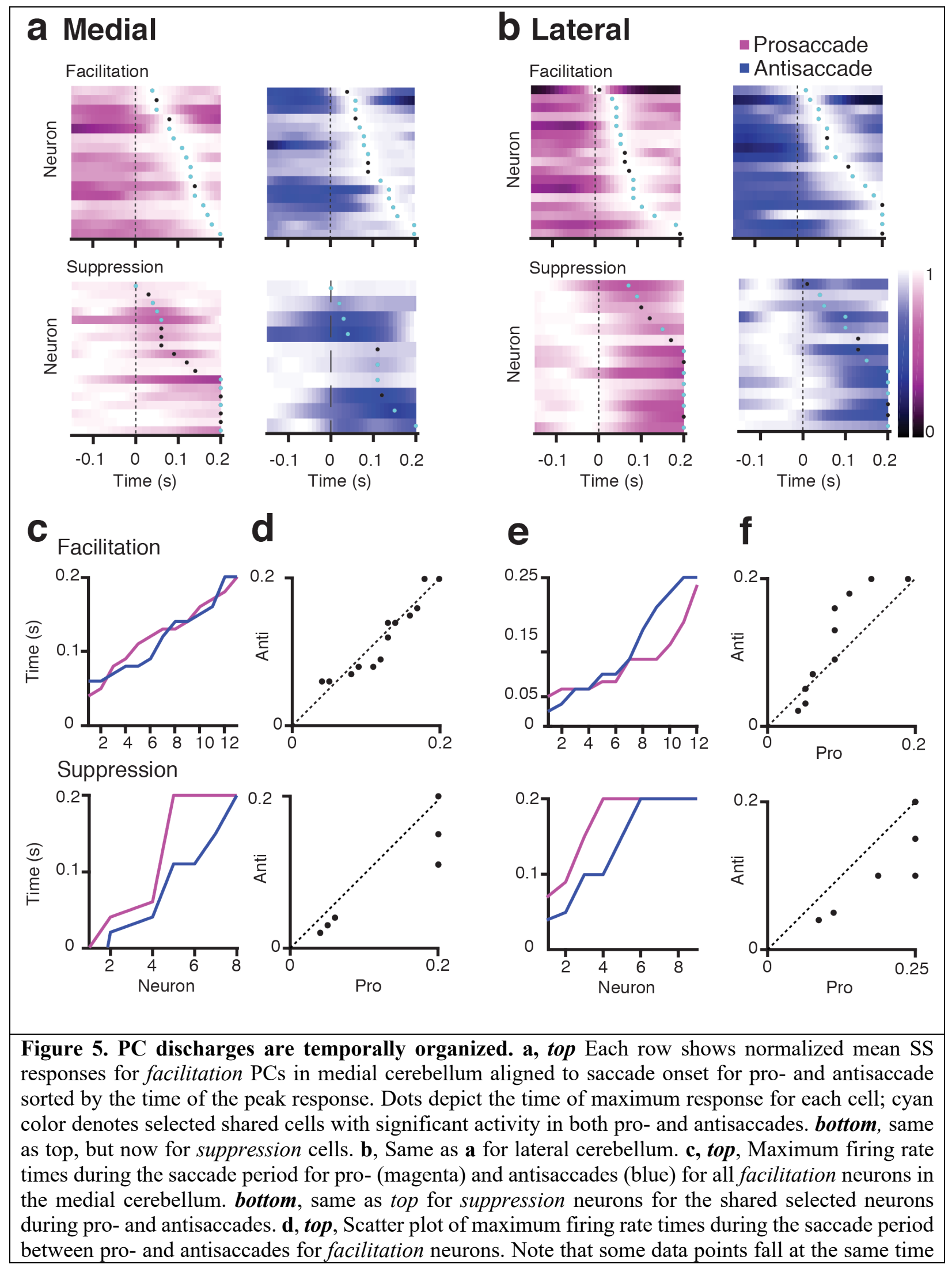


and will not be visible. bottom, same as top for suppression neurons. e and $\mathbf{f}$, Same as $\mathbf{c}$ and $\mathrm{d}$, but for lateral cerebellum.

Complex spike responses of PCs in lateral cerebellum show most prominent directional tuning and reciprocal firing

In a subset of recorded PCs, we were able to reliably isolate and analyze CS responses throughout the trials (Supplementary Table 1). We restricted the CS analysis only to cells with average CS firing rates higher than $0.5 \mathrm{~Hz}$ over the duration of the complete recording and at least 5 trials per direction for both prosaccades and antisaccades. Similar to the SS analyses, we examined the CS responses separately in the medial and lateral cerebellum during the instruction and execution periods (Fig. 6a-b). The average CS activities during execution $(1.11 \mathrm{~Hz} \pm 0.50$ s.d., $\mathrm{n}=11)$ and instruction $(1.10 \mathrm{~Hz} \pm 0.47$ s.d., $\mathrm{n}=11)$ in the medial cerebellum were not significantly different from those in the lateral cerebellum $(1.13 \mathrm{~Hz} \pm 0.52$ s.d., $\mathrm{n}=18$ and $1.15 \mathrm{~Hz} \pm 0.33$ s.d., $\mathrm{n}=18$, respectively, $p=0.36$ and $p=0.87$ for comparison medial versus lateral during execution and instruction, respectively).

The interaction between CS and SS responses was first quantified in the context of their directional preference during the execution period. Directional preference was determined by fitting a sinusoid to the CS and SS rates as a function of the direction of the saccade (Fig. 6 c-e). If the coefficient of determination $\left(\mathrm{R}^{2}\right)$ from the sinusoidal fit for CS responses was higher than 0.4, the cell was deemed directionally tuned and was included for further analyses (see Methods).

Figure 6c shows the direction preference of an exemplary neuron recorded in the medial cerebellum. We shifted the peak CS direction to 0 degrees and plotted the corresponding SS responses for the same neurons in the same way. When we did this for all the selected PCs of the 
medial cerebellum that were included based on their sinusoidal fits (5/38 pro- and 4/38 antisaccades), there seemed to be no clear pattern of significant relations between CS and SS responses in the context of directional modulation (Fig. 6d, Supplementary Table 3). We performed a Watson Williams test, a circular analog to the ANOVA, on the saccade directional tunings of the CS and SS responses of PCs in the medial cerebellum during prosaccades. We concluded that they were not exhibiting reciprocal modulation, since the test suggested that the directional tunings are not from different groups $(p=0.12, \mathrm{n}=5$, Fig. $\mathbf{6 g}$, left). The same was the case during antisaccades ( $p=0.91, \mathrm{n}=4$, Fig. 6g, right). Possibly, these findings obtained in the oculomotor vermis of the medial cerebellum reflect the low number of cells and/or the fact that the animals were to some extent overtrained, reducing the CS error signals to a minimum ${ }^{34,41}$. In contrast, in the lateral cerebellum, directional tuning and reciprocity were more prominent (Fig. 6b, 6e-f, and 6h). In the lateral cerebellum 12 and 9 out of 40 PCs showed significant saccade direction-sensitive modulation for pro- and antisaccades, respectively. Compared to medial PCs, the preferred directions of the CS responses of PCs in the lateral cerebellum were relatively often opposite to those of the SS responses (see Fig. 6e-f, for single neuron and population data, respectively). When comparing the directional preferences of the CS and SS responses by means of a Williams-Watson test this difference did hold for both pro- and antisaccades $(p=0.0014, \mathrm{n}=12$ and $p=0.006, \mathrm{n}=9$, respectively, Fig. 6h). Moreover, the vector averages of the CS and SS responses of PCs in the lateral cerebellum during prosaccades appeared opposite to those during antisaccades (note the flip of the orange and blue vectors in the left and right panel of Fig. 6h), highlighting their relevance for directional control of the saccades.

Next, we quantified the interaction between CS and SS responses during the instruction period (Fig. 6a and b, left panels). For every PC of both medial and lateral cerebellum with 
sufficient CS responses we calculated the maximum change in CS firing rate from baseline in the instruction window and the maximum change in SS firing rate from baseline in the $300 \mathrm{~ms}$ window around the time of the peak CS rate. Similar to the execution period, this was done for the pro- and antisaccade trials separately. When calculating the Pearson correlation coefficients between the peak CS and associated SS responses for the PCs in the medial cerebellum, no significant correlation was found $(\mathrm{R}=0.21, p=0.54$, for prosaccades; and $\mathrm{R}=0.15, p=0.67$ for antisaccades; $\mathrm{n}=11$ for both). However, when we applied the same procedure to PCs of the lateral cerebellum, a significant negative correlation between CSs and SSs was reached during the instruction period for prosaccade trials $(\mathrm{R}=-0.59, p=0.01 ; \mathrm{n}=18)$, but not during antisaccade trials $(\mathrm{R}=-0.21, \mathrm{p}=$ $0.41 ; \mathrm{n}=18)$. When we compared the reciprocity between the instruction and the saccade period, we observed that the cells in the lateral cerebellum that show reciprocity in the instruction period are the same ones that show reciprocity in the saccade period (Supplementary Figure 5). In line with previous findings ${ }^{49}$, this finding suggests that the cellular phenomenon of reciprocity can to some extent overrule the context-dependent nature of CS and SS encoding. Together, the data obtained during the instruction and execution of pro- and antisaccades suggest that the reciprocal modulation of CS and SS activity of PCs in the lateral cerebellum is more prominent than that in the medial cerebellum. 


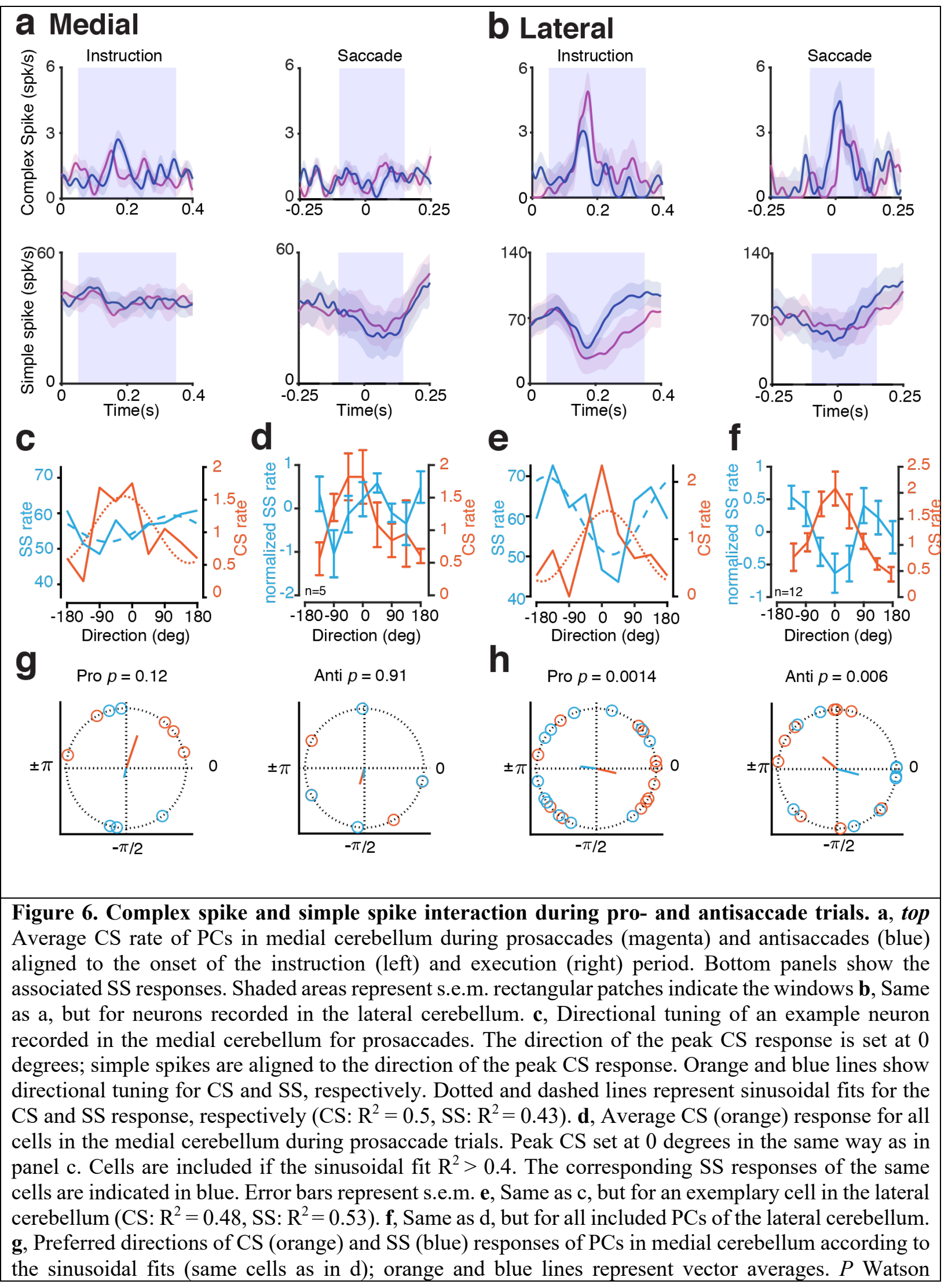


Williams test). h, Same as g, but for lateral cerebellum. Note that the PCs in the lateral cerebellum show a more pronounced level of reciprocity.

Facilitation and suppression Purkinje cells in medial and lateral cerebellum operate at different baseline frequencies

Unlike the SS responses of PCs of the suppression category, which showed a relatively late SS modulation during execution, those of the facilitation category showed a prominent increase in firing rate just before target appearance during the instruction period (see above). These data point towards dominant activities in downbound and upbound modules for the execution and instruction of saccades, respectively ${ }^{35}$. Given that downbound and upbound modules of PCs in the cerebellar cortex of rodents have been hypothesized to operate at a relatively high and low baseline SS firing frequency, respectively ${ }^{35}$, we next investigated whether the suppression and facilitation cells differed in this respect (see also ${ }^{42,50,51}$ ). We found that suppression cells indeed started at a relatively high baseline SS firing frequency in both the medial and lateral cerebellum $(62.43 \pm 3.76$ spks/s and $69.6 \pm 6.12 \mathrm{spks} / \mathrm{s}$, respectively), and that facilitation cells started at a significantly lower baseline SS firing frequency in both regions $(56.36 \pm 3.65 \mathrm{spks} / \mathrm{s}$ and $54.72 \pm 3.76 \mathrm{spks} / \mathrm{s}, p$ $=0.02$; one-tailed Wilcoxon rank-sum test comparison between all facilitation vs suppression cells pooled in both areas). Together, these data imply that PCs of non-human primates may follow the same learning rules as those proposed for rodents ${ }^{52}$. 


\section{Discussion}

The antisaccade task has served to investigate hubs of flexible behavioral control and their interaction, where a correct suppression of a prosaccade followed by a response to the opposite direction forms a feature of executive control ${ }^{4,53}$. We found evidence for cerebellar involvement in volitional control of this behavior in both medial (i.e., OMV) and lateral (i.e., crus I/II) cerebellum by recording the activity PCs during pro- and antisaccades in non-human primates. Our findings add to the growing body of evidence pointing to an important contribution of the cerebellum to cognitive processes in general and voluntary behavior in particular. We demonstrated that PCs in both areas modulate their activity depending on the trial type, and that SS responses of populations of PCs in the lateral cerebellum are able to discriminate the type of trial. Even though the lateral cerebellum is not traditionally viewed as a saccade-control area, our findings support early reports where saccades are elicited following electrical stimulation to lateral regions of the hemispheres, especially in crus $\mathrm{I} / \mathrm{II}^{54}$.

Antisaccades exhibit saccade kinematic properties that differ from standard prosaccades, a result consistent with previous reports in human and non-human primates ${ }^{2,46}$. Even so, the preferential modulations of both SS and CS responses in the PCs were not highly correlated to any of those kinematic changes (Supplementary Figure 1). This suggests that the observed neuronal activity was not merely an efference copy of the motor command. Specifically, we argue that the observed activity during the instruction period reflects the preparatory or planning phase of the voluntary movement in which the PC firing is determined by the cue and that this encoding may be particularly relevant for the facilitation cells. This is in line with recent findings in cerebellar atrophy patients who show impairments when making goal-oriented movements ${ }^{55}$. 
Various parameters of PC activity differed between medial and lateral cerebellum. SS activity of PCs in the lateral cerebellum (1) showed a greater firing rate during the execution of saccades, (2) exhibited more prominent saccade-related signals at the population level, and (3) displayed more signal separation/selectivity for pro- and antisaccades during the instruction period. Likewise, their CS activity showed a higher level of reciprocity with respect to simple spike activity, during both the execution and instruction period. In contrast, PCs in the medial cerebellum were more sensitive to trial history, and those of the suppression category showed a relatively early SS modulation during execution of both pro- and antisaccades. Together, these data indicate that different modules of different cerebellar regions can both contribute to the same complex behavior, yet with different propensities.

\section{SS and CS modulation during instruction period}

Studies of the eye movements from patients with cerebellar lesions suggest that the role of the cerebellum might not be in the initiation of saccades, but in the correct and accurate execution of them ${ }^{56,57}$. Our results indicate that PCs in lateral cerebellum receive information about the trial identity. Although only a small fraction of neurons in Crus I/II exhibited significantly different SS activity between both conditions, we showed that these contain sufficient information to distinguish a pro- from an antisaccade (Fig. 3 and Fig. 4). This is in part supported by a previous study showing that the posterior part of the dentate nucleus fires more during the preparation of antisaccades and that local inactivation promotes more antisaccade errors ${ }^{17}$. This instructionrelated activity in antisaccade trials is thought to be relayed to the superior colliculus (SC) and related nuclei in the brainstem that are engaged in curtailing reflexive saccades ${ }^{58}$. Together with the existing data on the dentate nucleus and its downstream structures, the persistent SS modulation 
of PCs in the lateral cerebellum upon cue presentation during the instruction period suggests that they may maintain and update an internal model for well-timed activity during motor planning ${ }^{21}$. This in turn suggests that this part of the cerebellum, through its projections to the prefrontal cortex, participates in optimizing not only the execution of saccades, but also the preparation thereof.

Correct decoding of a visual stimulus is an essential component of the pro- and antisaccade task. Visual responses in the cerebellum have been reported in several areas including the floccular complex, crus I/II and the inferior semilunar lobe (VII/VIII) ${ }^{59}$. Our results of PCs in the lateral cerebellum show direct CS signals timed to the onset of the visual cue related to the correct execution of the subsequent pro- or antisaccade. Many cells in this region showed a CS drive during both the instruction and execution epoch, complementing the findings that their CS-SS reciprocity and simple spike modulation are also prominently spread throughout the task. Dentate nucleus neurons have activity bridging the period from task instructions to motor execution ${ }^{17}$, which could be derived from the same population of PC's as we recorded in the lateral cerebellum. The results from our current study raise the possibility that CS responses play a similar role in decoding of visual stimuli for the preparation of ensuing movements.

Contemporary models of antisaccade decision-making require evidence accumulation to reach a decision boundary in the frontal cortex ${ }^{2}$. The PCs in the cerebellum receive an enormous amount of diverse sensory and motor information through their parallel fiber inputs, which may also facilitate efficient evidence accumulation. Classical models of cerebellar function dictate that PCs pick relevant parallel fiber inputs to drive SS firing through means of climbing fiber inputs, which cause plasticity at the parallel fiber to PC synapse ${ }^{60}$. The PCs that we measured in the lateral cerebellum carry both SS and CS signals during the instruction period of the antisaccade task. We hypothesize that through the pre-learned associations of the right parallel fiber input based on the 
CS, relevant evidence from the stimulus (i.e., dot color in instruction) for the selection of the required action (i.e., pro- or antisaccade) can be rapidly relayed from the lateral cerebellum to the frontal saccade areas (e.g., SEF, FEF, DLPFC). In cerebellar patients, latencies for antisaccades are prolonged, indicating a less efficient decision-making process in absence of cerebellar input ${ }^{55}$. When the decision is made in the cortical areas, projections from FEF to the SC and OMV of the medial cerebellum will generate a fast and accurate execution of the eye movement.

\section{SS and CS modulation during saccade execution}

Single PCs modulated their SS activity during the execution of pro- and antisaccades in both medial and lateral cerebellum, but only lateral cerebellar PCs were able to successfully discriminate the type of trial. Despite the heterogeneity of the responses, neurons that facilitated or suppressed their response after saccade onset exhibited differential activity during both conditions (Fig. 3). As a population, PC responses were different when executing a saccade towards a visible target than when using an internally generated goal. This is particularly interesting for two reasons: Firstly, the medial cerebellum has always been identified as a motor and timing controller for the different types of saccades, interacting with cortical and brainstem brain areas ${ }^{61-63}$, while the role of the lateral cerebellum in saccade control has remained under debate. Indeed, lesions to the lateral cerebellum can delay onsets of saccades between 10 and 60 $\mathrm{ms}$ and result in variable hypo- and hypermetria ${ }^{64}$, but the contribution of Purkinje cell activity in the lateral cerebellum to proactive control of saccades and flexible behavioral in general has not been conclusively proven. Secondly, the differential cerebellar activity during antisaccades implies that it contains neural signatures to modulate preparation and execution by encoding information about the current context of the upcoming action (saccade), which is necessary for the fast classification of the response as correct or erroneous. This finding is in line with the impact of 
focal cerebellar lesions in humans on the changes in potentials observed during performance monitoring in an antisaccade $\operatorname{task}^{16}$.

The CS responses in the medial cerebellum have been shown to encode errors in movement execution, enabling PCs to correct errors in subsequent movements by pruning SS responses ${ }^{65}$. Unlike prosaccades, the antisaccades do not have a visual target. Therefore, keeping them accurate is more challenging, which is displayed in the broader range of saccade amplitudes in Figure 1. Furthermore, antisaccade accuracy monitoring depends on internal estimates of saccade amplitudes, which could explain why adaptation of antisaccades poorly transfers to prosaccades ${ }^{66}$. Taking this into account it seems most likely that antisaccade amplitude is monitored upstream of the medial cerebellum ${ }^{67}$.

In line with other studies on CS responses of PCs in lateral cerebellum during visual pursuit, we found that they also modulate during saccades ${ }^{59}$. Similar to the SSs, their activity is modulated depending on the trial-type. In the saccade period, the CS activity in the lateral PCs shows direction selectivity similar to that observed in the medial cerebellum ${ }^{59}$. This implies that the saccade-related CS activity found in the lateral cerebellum is not only involved in the preparation of the motor response, but also in its execution. To what extent well-timed CS synchrony in the lateral cerebellum, which can contribute to coordination of complex movements ${ }^{68,69}$, also contributes to the generation of pro- and antisaccades remains to be shown.

\section{Cerebellar modules operate in parallel}

The data on suppression PCs in the medial cerebellum showing a relatively late SS modulation during execution of both pro- and antisaccades as well as those on facilitation PCs in the lateral cerebellum showing a prominent modulation at the end of the instruction of antisaccades 
indicate that different modules of different cerebellar regions can simultaneously contribute to the same complex behavior, yet with different particularities. A similar conclusion was drawn from a recent study on delay eyeblink conditioning ${ }^{70}$. Even though this form of conditioning is classically considered to be controlled solely by modules in lobule simplex of the lateral cerebellum ${ }^{35}$, Wang and colleagues have shown that modules of the medial cerebellum are equally essential, yet also contributing in a slightly differential fashion, possibly regulating mainly muscle tone ${ }^{70}$. Interestingly, similar to the current pro- and antisaccade tasks, during eyeblink conditioning the medial and lateral cerebellum also both engage facilitation and suppression cells, and they also operate at relatively low and high baseline firing frequencies, respectively ${ }^{38,70}$. Our current finding that facilitation and suppression cells appear to play a more dominant role during the instruction and execution of the saccade task, respectively, further highlights the differential functional relevance of the upbound and downbound modules ${ }^{35}$.

Previous reports of activity in the dentate nucleus, which is the main target of PCs in the lateral cerebellum, have shown mixed evidence regarding the direction of modulation during saccade-related behavior ${ }^{25,71}$. Kunimatsu and colleagues found only upward modulating units during self-initiated saccades ${ }^{71}$, whereas Ashmore and Sommer revealed groups of both facilitating and suppressing cells during delayed saccades ${ }^{25}$. Possibly, these differences reflect differences in the behavioral paradigms and/or the different time windows analyzed. In our dataset, the modulation exhibited by PCs is bidirectional in both medial and lateral cerebellum with populations of both facilitation and suppression cells. 
PC activity in both medial and lateral cerebellum displays stable time-ordered sequences

Sequence activation of neurons is a feature observed in different brain areas. Typically, neurons exhibit their maximum response at discrete time points during structured behaviors, often reflecting the connectivity of the network ${ }^{43,44}$. This also holds for the cerebellum, where the sequences of activity may serve to enhance motor timing ${ }^{21,24,40}$. Here, we observed structured sequenced SS responses in both medial and lateral cerebellum during both types of saccades. These sequences could be interpreted as a mechanism to control the timing of eye movements, including their duration and the moment of their peak amplitude. Lesions of medial and lateral cerebellum are in line with this notion ${ }^{64}$. In addition, these sequences could represent a distributed computational resource to aid in saccade execution. To test this hypothesis future experiments need to perform PC recordings while the monkeys learn the pro- and antisaccade task to observe the formation of sequences during the training of the task ${ }^{34}$. 


\section{Methods}

\section{Animals}

All procedures complied with the NIH Guide for the Care and Use of Laboratory Animals (National Institutes of Health, Bethesda, Maryland), and were approved by the institutional animal care and use committee of the Royal Netherlands Academy of Arts and Sciences. Two adult male rhesus non-human primates (Macaca mulatta), Mi and Mo, were used in this study.

\section{Surgical procedures}

Animals were prepared for eye movement and awake, extracellular single unit recordings in the cerebellum using surgical and electrophysiological techniques in a two-step procedure (Roelfsema et al., 2012). Under general anesthesia induced with ketamine (15 mg/kg, i.m.) and maintained under intubation by ventilating with a mixture of $70 \% \mathrm{~N} 2 \mathrm{O}$ and $30 \% \mathrm{O} 2$, supplemented with $0.8 \%$ isoflurane, fentanyl $(0.005 \mathrm{mg} / \mathrm{kg}$, i.v. $)$, and midazolam $(0.5 \mathrm{mg} / \mathrm{kg} \cdot \mathrm{h}$, i.v. $)$, we first implanted a titanium head holder to painlessly immobilize the monkey's head. Four months later, once the monkeys had mastered the behavioral task (see below), a custom-made $40 \mathrm{~mm}$ chamber was implanted under the same anesthesia conditions as described above to gain access to the cerebellum with a 25 angle (Fig. 1b). Animals recovered for at least 21 days before training was resumed. 


\section{Behavioral task}

Animals were trained to perform a randomized interleaving pro- and antisaccade task in 8 different directions (cardinal and diagonal directions), with amplitudes between $5^{\circ}$ and $14^{\circ}$ (Fig. 1a). All recordings were conducted with the monkey in complete darkness. During training and experiments, monkeys were seated in a primate chair (Crist Instrument, USA) with their head restrained at $100 \mathrm{~cm}$ from a screen with a resolution of $1,024 \times 768$ pixels. Visual stimuli were presented by a CRT-Projector Marquee 9500 LC (VDC Display Systems, Florida, USA) with a refresh rate of $100 \mathrm{~Hz}$. Binocular vision was unrestricted. A trial started when the monkey fixated on a red or green fixation point at the center of the screen for a random time between 300-500 ms. Next, a red target appeared in one of 8 different target locations. After $100 \mathrm{~ms}$ the fixation point changed to gray and the monkey had to perform either a prosaccade toward the target (when fixation point was red) or antisaccade in the opposite direction with the same amplitude as the target, (when fixation point was green) within $500 \mathrm{~ms}$. Colors of fixation point and target were changed to blue and magenta in the figures to accommodate colorblind readers.

The animals received liquid reward if they performed a correct saccade (within $6^{\circ}$ of the (anti-)target) and maintained fixation for $100 \mathrm{~ms}$. Within a single block the 8 (targets) * 2 (pro/anti) possible configurations were presented in a random order.

\section{Recordings}

Position of the right eye was recorded with an infrared video-based eye tracker (iViewX Hi-Speed Primate, SMI GmbH, Germany) at a sampling rate of $350 \mathrm{~Hz}$. The eye tracker was calibrated before every recording session by having the animal look at $1^{\circ}$ target grid consisting of 9 points (one at the center of the screen and 8 points $10^{\circ}$ apart) to adjust offset of $\mathrm{X}$ and $\mathrm{Y}$ position 
by hand. Pre and post-surgical MRI-images were used to build a 3D model of the skull and cerebellum for anatomical localization of cerebellar oculomotor vermis (lobules VI and VII) and lateral cerebellum (crus I/II, Fig.1b) ${ }^{72}$. Single-unit recordings were obtained using tungsten glasscoated electrodes (1-2 M $\Omega$, Alpha Omega Engineering, Nazareth, Israel) through a 23-gauge guide tube, which was inserted only through the dura. A motorized microdriver (Alpha Omega Engineering, Nazareth, Israel) with a 1-mm spaced grid was used to introduce the electrode and map the recording sites with a maximum resolution of $0.25 \mathrm{~mm}$.

Extracellular recordings were digitized and sampled at $44 \mathrm{kHz}$ and subsequently stored during the experiment using a Multi-Channel Processor (Alpha Omega Engineering, Nazareth, Israel). Single units were determined to be a Purkinje cell by the presence of spontaneous firing of simple spikes (SS) and complex spikes (CS) as well as the presence of a concurrent short pause in SS firing following a CS (i.e. climbing fiber pause) ${ }^{73}$, as discriminated online using Multi-Spike Detector (MSD, Alpha Omega Engineering). The discovery of a saccade-related site was often guided by the modulating multi- unit signals generated by granule cells during saccades. Trial history, horizontal and vertical eye position and electrophysiological data were concurrently stored for offline analysis.

\section{Data Analysis Software}

All analyses were performed off-line using custom programs written in MATLAB (The Mathworks, Natick, MA, USA).

Eye movement analysis 
Eye position was sampled at $350 \mathrm{~Hz}$ with an infrared video eye tracker which tracked the pupil center of mass. Noise was reduced using a finite impulse response filter and a savitzky-golay filter (20 ms window). Eye velocity and acceleration traces were created by differentiating the signal. Eye acceleration was further processed using a median filter. Saccade onset and offset were detected using an adaptive threshold based on 6 s.d. of the noise during fixation from the recording session of that day as described in Nyström and Holmqvist $(2010)^{74}$. Trials with reaction times less than $80 \mathrm{~ms}$ were excluded, as these were thought to represent anticipatory actions.

\section{Electrophysiological analysis and statistics: Simple spikes}

In order to perform statistical analysis, neurons were required to have at least 10 recorded trials per direction. Note that only correct trials and saccade-related neurons were incorporated in the analyses. This was due to the fact that both monkey Mo and Mi exhibited an expert level performance reaching $>90 \%$ correct responses. Therefore we lacked statistical power to analyze the incorrect trials. A neuron was considered to be saccade-related when SS firing during the baseline period, defined as a window 400 to $50 \mathrm{~ms}$ before trial onset in the intertrial interval was significantly different from the saccade execution period activity defined to be $150 \mathrm{~ms}$ after saccade onset (Wilcoxon rank test; $p<0.05$ ) for at least one of the 8 directions. We then computed the instantaneous SS firing rate of the neurons using a continuous spike density function (SDF) generated by convoluting the spike train with a Gaussian function of $\sigma=50 \mathrm{~ms}$ width and averaging all the individual spike density functions ${ }^{75,76}$. We compared the number of spikes for all pro- and antisaccade trials of each PC using a Kolmogorov-Smirnov test which determined if the activity of a PC was significantly different between a pro- and antisaccade with a $p$ value $<0.05$. The characteristics of the saccade-related activity, as previously reported in PC recordings, was 
quite heterogeneous. On that account, we categorized the PCs if their activity increased after instruction or saccade onset as facilitation or decreased as suppression. For the instruction period, we compared the mean firing rate of each cell from a window $400 \mathrm{~ms}$ before instruction onset to a period of $300 \mathrm{~ms}$ at the end of the instruction period. For the saccade period we compared the mean firing rate of each cell from a window $150 \mathrm{~ms}$ before saccade onset to a window from saccade onset to $150 \mathrm{~ms}$ after saccade onset (Wilcoxon signed rank, $p$ value of $<0.05$ ). To determine the correlation between kinematic parameters and neuronal activity we determined Pearson's correlation coefficient $(r)$ for all neurons in the two tasks. Modulation ratio for both areas was obtained by computing the ratio between the response during pro- and antisaccades.

Change in firing rate during the instruction period was computed by taking the difference between the mean firing rate during the baseline period separate for pro- and antisaccade trials. For the saccade period, we compared the mean activity $150 \mathrm{~ms}$ before the saccade onset to a period $150 \mathrm{~ms}$ after saccade onset.

To compare the dynamics in the temporal responses of PCs, we trial-averaged the responses during the saccade period, normalized and sorted the activity of each PC by their maximum response using half of the trials. Figure 5 is plotted using the other half of the trials. To test that the time of the responses was not an observation by chance, we randomly picked $50 \%$ of the trials, and plotted their maximum firing rate times. We repeated this procedure 100 times. To test whether the neurons change substantially their maximum firing location, we classified their position as 1 or 0 if they fired maximally in one of 4 windows around saccade onset: -150 to -75 ms, - -75 to $0 \mathrm{~ms}, 0$ to $75 \mathrm{~ms}, 75$ to $150 \mathrm{~ms}$ (Supplementary Figure 3). Similarity index between all and redrawn trial's maximum location times was computed by estimating the cosine similarity between strings of binary classification patterns $\left(\mathrm{B}_{i}\right.$ and $\left.\mathrm{B}_{\mathrm{j}}\right)$ obtained by comparing two windows 
at a time (all trials vs. randomly drawn). Similarity index: $\cos (\theta)=\frac{\left(B_{i} \cdot B_{j}\right)}{\|\left(B_{i}\|\|\left(B_{j} \mid\right.\right.}$. Identical classification patterns have an index of 1.

To test how well a single PC can discriminate between a pro- or antisaccade we computed a binomial proportion test over time. This will show if a PC contains information about the trial condition and the time points at which the discrimination is more pronounced. We first computed the probability of a spike in every trial in every condition in bins of $10 \mathrm{~ms}$. Then, we computed a binomial proportion test: $Z=\frac{\widehat{p_{1}}-\widehat{p_{2}}}{\sqrt{\hat{p}(1-\widehat{p})\left(\frac{1}{n_{1}}+\frac{1}{n_{2}}\right)}}$, where $\hat{p}_{1}$ and $\hat{p}_{2}$ is the probability of a spike in a proand antisaccade respectively: $\hat{p}=\frac{n_{1} \widehat{p_{1}}+n_{2} \widehat{p_{2}}}{n_{1}+n_{2}}, n_{1}$ is the number of trials in prosaccades and $n_{2}$ is the number of trials of antisaccade trials.

To test if the identity of previous trials had an effect on the current trial $(n)$, we computed the absolute difference in SS between the current trial $n$ and one previous trial $(n-1)$ or two previous trials $(n-2)$. We first identified all correct trials where all possible combinations presented in at least 10 repetitions and computed the absolute difference in SS activity for all cells in medial and lateral cerebellum (Kruskall-Wallis test).

\section{Electrophysiological analysis and statistics: Complex spikes}

Cells were included into the analysis for complex spikes if they had a CS firing rate of between 0.5 and $2 \mathrm{hz}$ over the entire recording session and at least 5 trials for every of the 8 pro and antisaccade directions. To determine if the CS modulated to the one of the task epochs, we calculated the baseline firing rate \pm 3 standard deviations. Baseline firing was considered a period of $500 \mathrm{~ms}$ of inter-trial activity. When the CS rate exceeded this upper or lower limit during either 
the instruction or the saccade window (50 - $350 \mathrm{~ms}$ after onset of the instruction and -150 to +150 ms around the onset of the saccade) the unit was considered as modulating.

To determine if cells had any directional modulation a sinusoid was fitted on the SS and CS firing rate in the saccade window as a function of the direction using formula: $Y=a * \sin (b *$ $x+c)+d$. When fitting, $\mathrm{b}$ was fixed at 1 so that only one period was fitted. The phase shift found as c was used in figure $6 \mathrm{~g}$, h to compare preferred directions. If the $\mathrm{R}^{2}$ of the sinusoidal fit for was higher than cutoff value 0.4 the cell was deemed direction selective. To avoid constraining the analysis too much, we included the cells in further analysis of directional reciprocity if the cell had CS directional selectivity. Cells were aligned to their preferred CS direction and the Pearson correlation coefficients were calculated to determine if there was directionally dependent reciprocity between SS and CS rates in the different groups of cells. Lastly to determine if there was a difference between CS and SS directionality the Watson Williams test was used on the unaligned CS and SS directions to determine if they were from different groups.

\section{Acknowledgements}

We thank Kor Brandsma and Anneke Ditewig for their excellent biotechnical assistance; Beerend Winkelman for providing the MATLAB code for spike sorting; Chris van der Togt for providing assistance with stimulus presentation; Mario Negrello for providing the MATLAB code for saccade detection; Geert Springeling, Peter Thier, Peter W. Dicke, Ruud Smith for their help with anatomical scans and 3D model building; and Kaushik J. Lakshminarasimhan for helpful comments in the analysis. This research was supported by FP7-C7 European Commission, the Marie Curie Initial Training Network ITN-GA-2009-238214, ENW-Klein, ZonMw, Netherlands 
Organization for Scientific Research, European Research Council (Advanced Grant, and Proof of

Concept Grant), Medical NeuroDelta Programme, Topsector Life Sciences \& Health (Innovative

Neurotechnology for Society or INTENSE), and the Albinism Vriendenfonds Netherlands Institute for Neuroscience.

\section{Author contributions}

CIDZ, AB, MF, NF and EA designed the study and analysis. EA and PR performed surgeries on the animals. EA and NF executed the experiments and EA, NF, AB and PJH analyzed the data. EA, NF, AB and CIDZ wrote the first draft. All authors edited the manuscript.

\section{Competing interests}

The authors declare no competing interests.

\section{Data and code availability}

The data that support the findings of this study and code for analysis of in vivo eye movement and Purkinje cell recordings are available from the corresponding author upon reasonable request. 
bioRxiv preprint doi: https://doi.org/10.1101/2021.03.26.437236; this version posted March 28, 2021. The copyright holder for this preprint (which was not certified by peer review) is the author/funder, who has granted bioRxiv a license to display the preprint in perpetuity. It is made available under aCC-BY-NC-ND 4.0 International license.

\section{Supplementary Figures}




\section{a Medial}

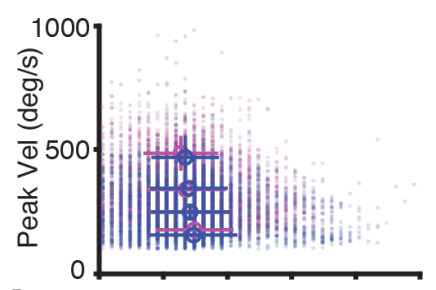

b

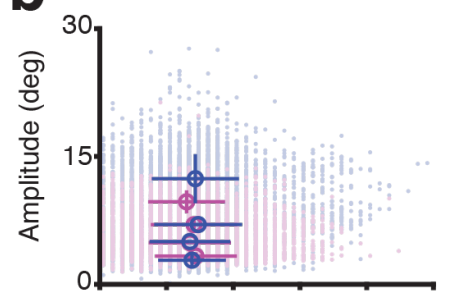

C

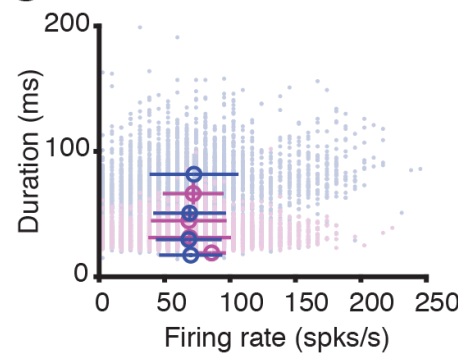

d

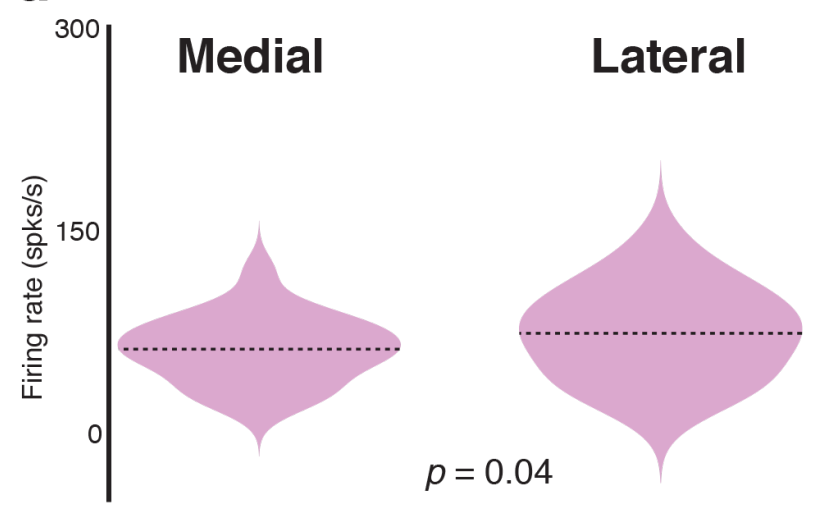

Lateral
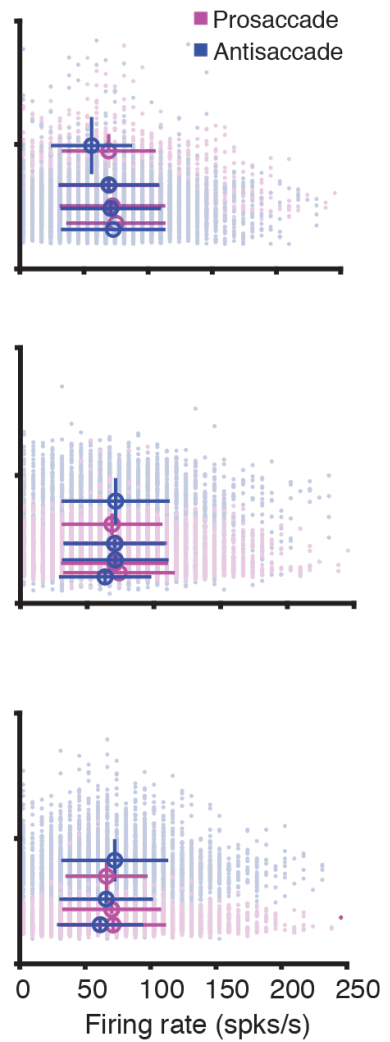

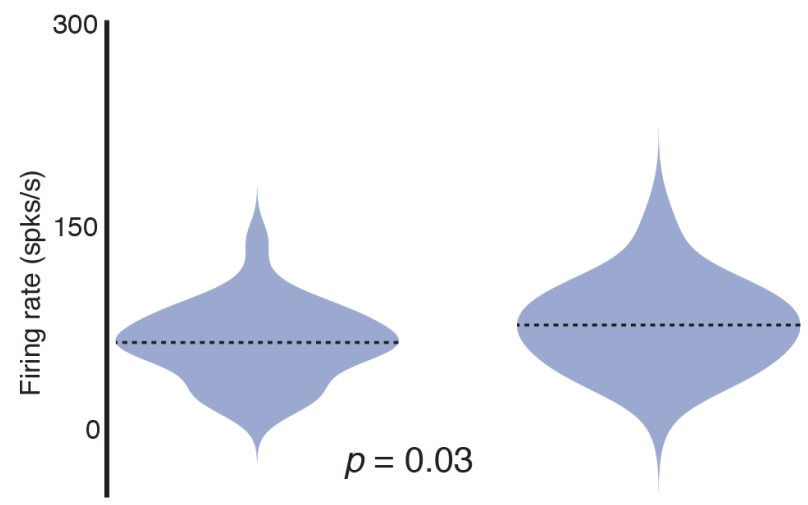


Supplementary Figure 1. PCs in medial (left) and lateral cerebellum (right) modulate similarly with
saccade parameters, but fire differently during pro and antisaccades . a, Amplitude (deg). b, Peak velocity. c, Duration for all saccades (correct and incorrect) plotted against simple spike firing rates for all trials in pro- (magenta) and antisaccades (blue). Open circles show the firing rate mean and s.d. for 4 different predetermined blocks in each saccade kinematics: (a) 4, 6, 8, and $>8 \mathrm{deg}$, (b) 200, 300, 400, and $>400 \mathrm{deg} / \mathrm{s}$, (c) 20,40,60, and $>60 \mathrm{~ms}$. Pearson correlations for medial cerebellum: Amplitude pro- $r=-$ 0.05 , anti- $r=0.04$; duration pro- $r=0.007$, anti- $r=0.14$; and peak velocity pro- $r=0.11$, anti- $r=-0.08$. Pearson correlations for crus-I/II: Amplitude pro- $r=0.01$, anti- $r=-0.04$; duration pro- $r=0.005$, anti- $r$ $=-0.05$; and peak velocity pro- $r=-0.01$, anti- $r=-0.03$. Error bars denote s.e.m. d, Violin plots show the mean average firing rate for all cells during prosaccades (top) and antisaccades (bottom) for medial (left) and lateral (right) cerebellum. Dashed lines depict the mean value for the population. The p values in the figure are for a Wilcoxon rank sum test for each condition comparison between medial vs lateral cerebellum. 


\section{a Medial}
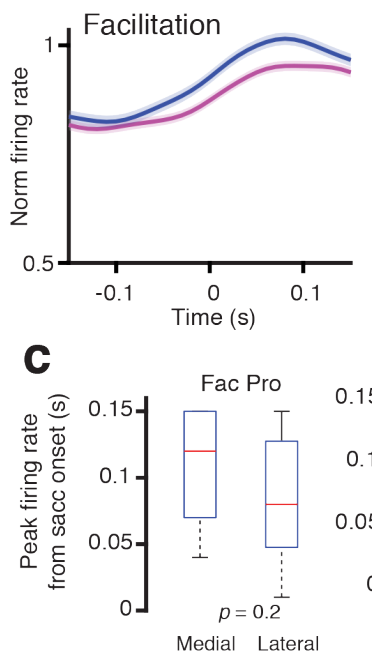

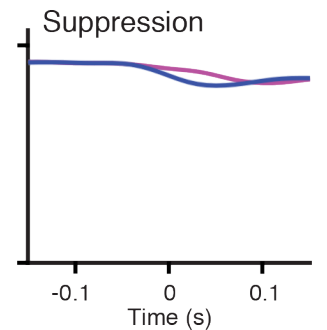

\section{b Lateral}
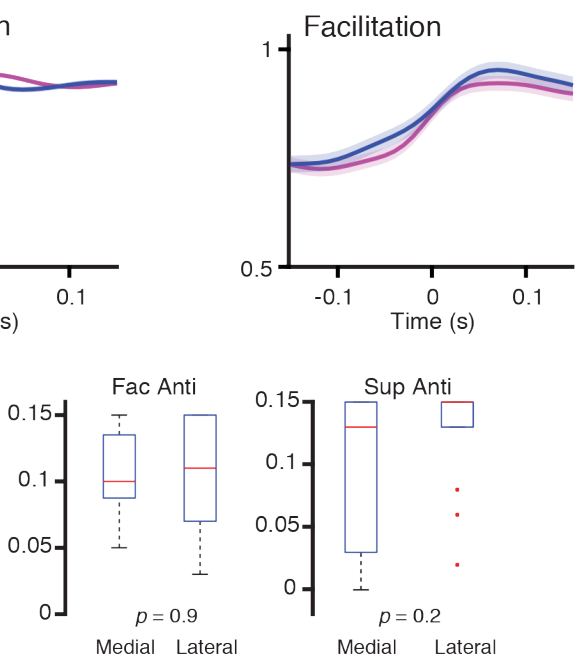

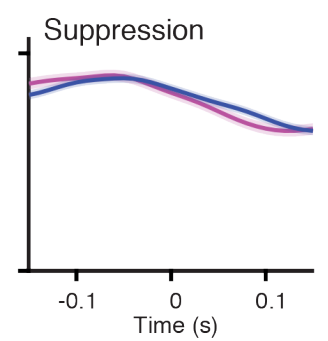

Supplementary Figure 2. Time-course of responses for different types of activity. a, Time-course of response averaged for all neurons in the medial cerebellum that either increased their firing during both conditions (facilitation) or decreased it (suppression) after the instruction onset. Shaded regions denote s.e.m. b, Same as a for lateral cerebellum. c) Time of peak responses separate by the type of activity exhibited by PCs in medial and lateral cerebellum. 

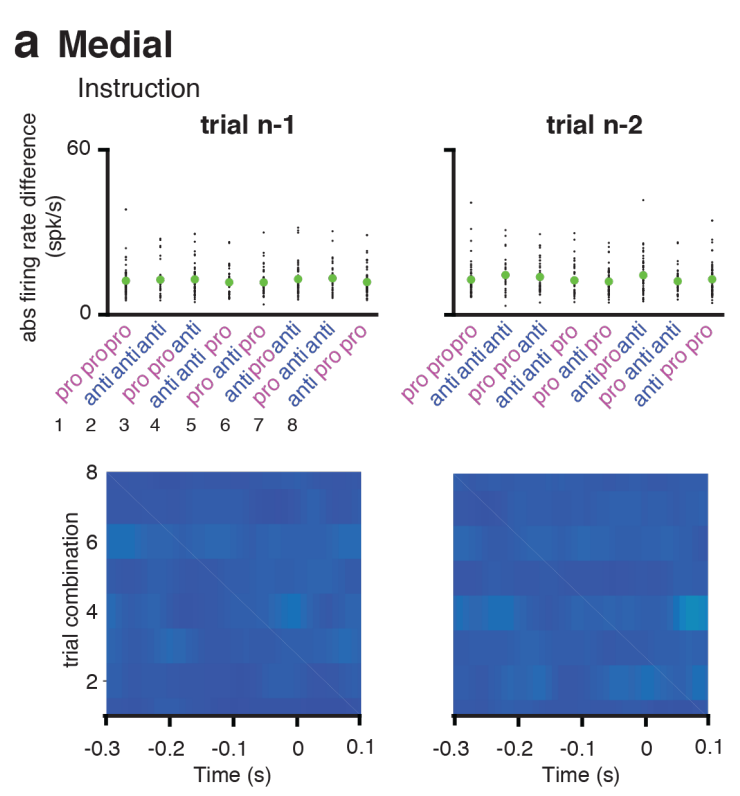

\section{Lateral}
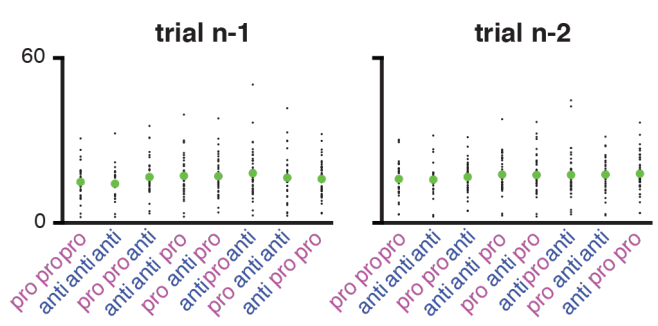

b
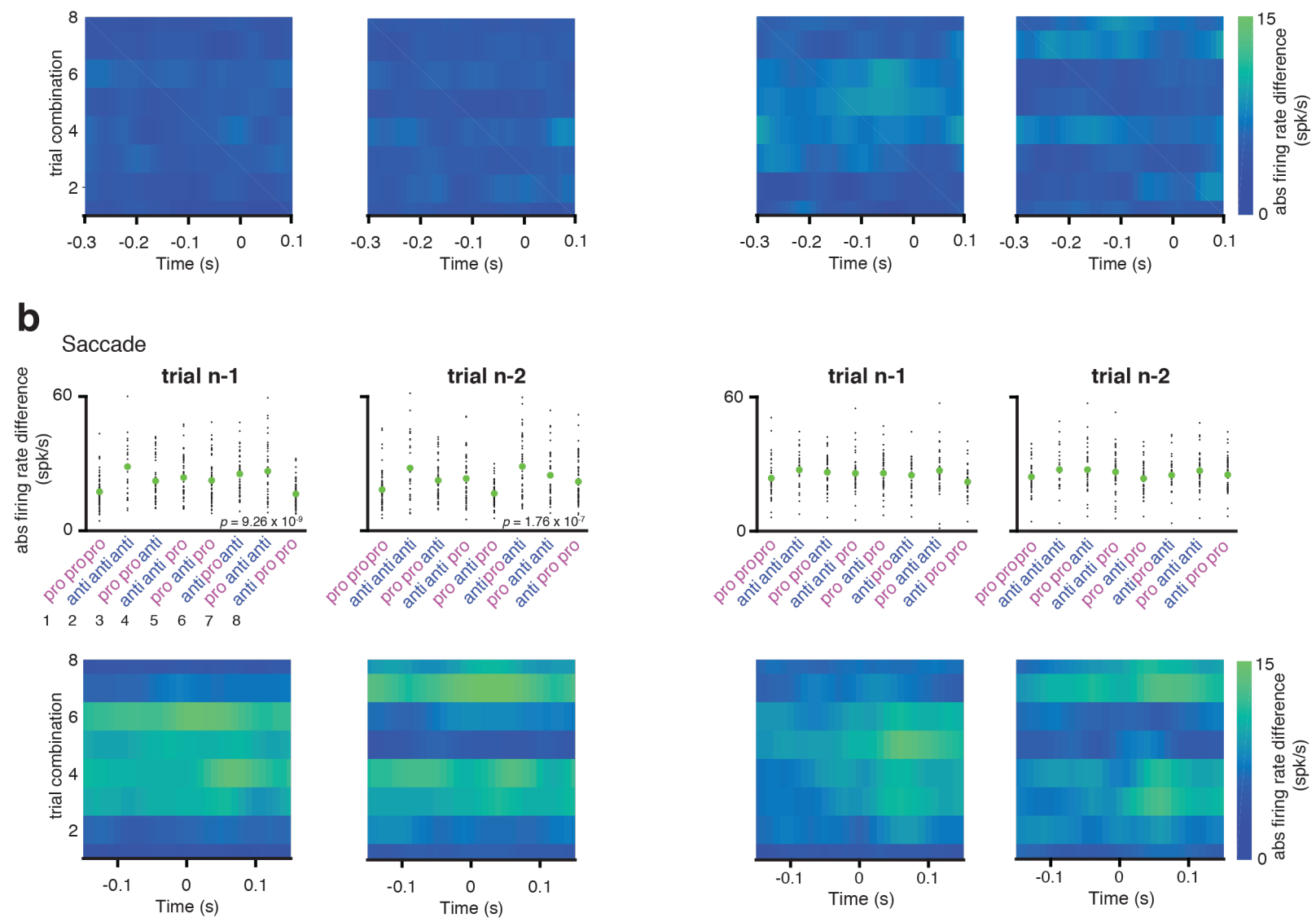

\section{Supplementary Figure 3. Trial history modulates PC activity of medial cerebellum during execution} (i.e., saccade) period. a top, Absolute difference in spiking activity during the instruction period for trial $n$ compared to one trial back (trial $n-1)$ and two trials back (trial $n-2)$ for all cells in medial ( $\mathrm{n}=81$; left) and lateral cerebellum $(\mathrm{n}=68$; right). Green dots depict mean activity. Inset shows $p$-value for a Kruskal-Wallis test. a bottom, Heat maps show absolute firing difference estimated between the PSTH in trial $n$ and trial $n-1$ and $n-2$ for every combination. b, Same as (a) for the saccade period. 


\section{a Medial}
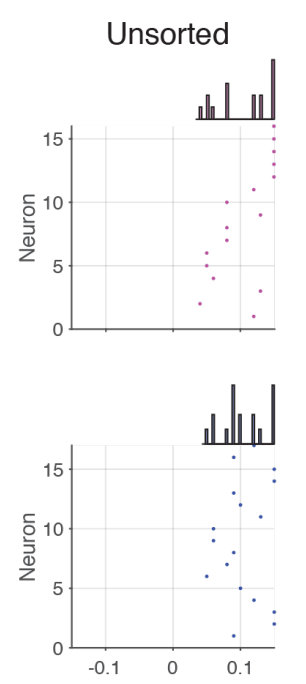

\section{b Lateral}

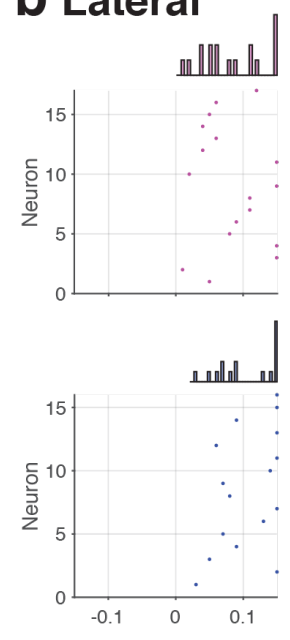

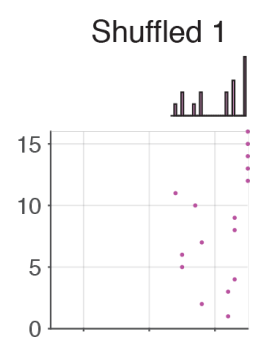
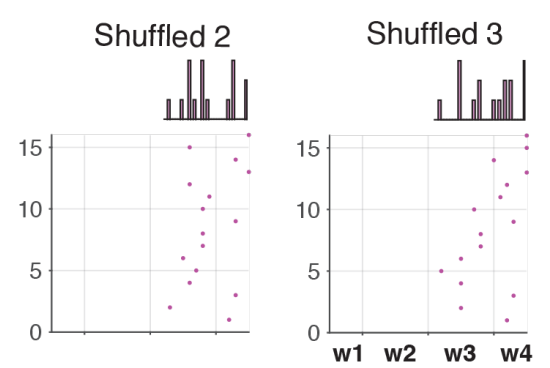

C
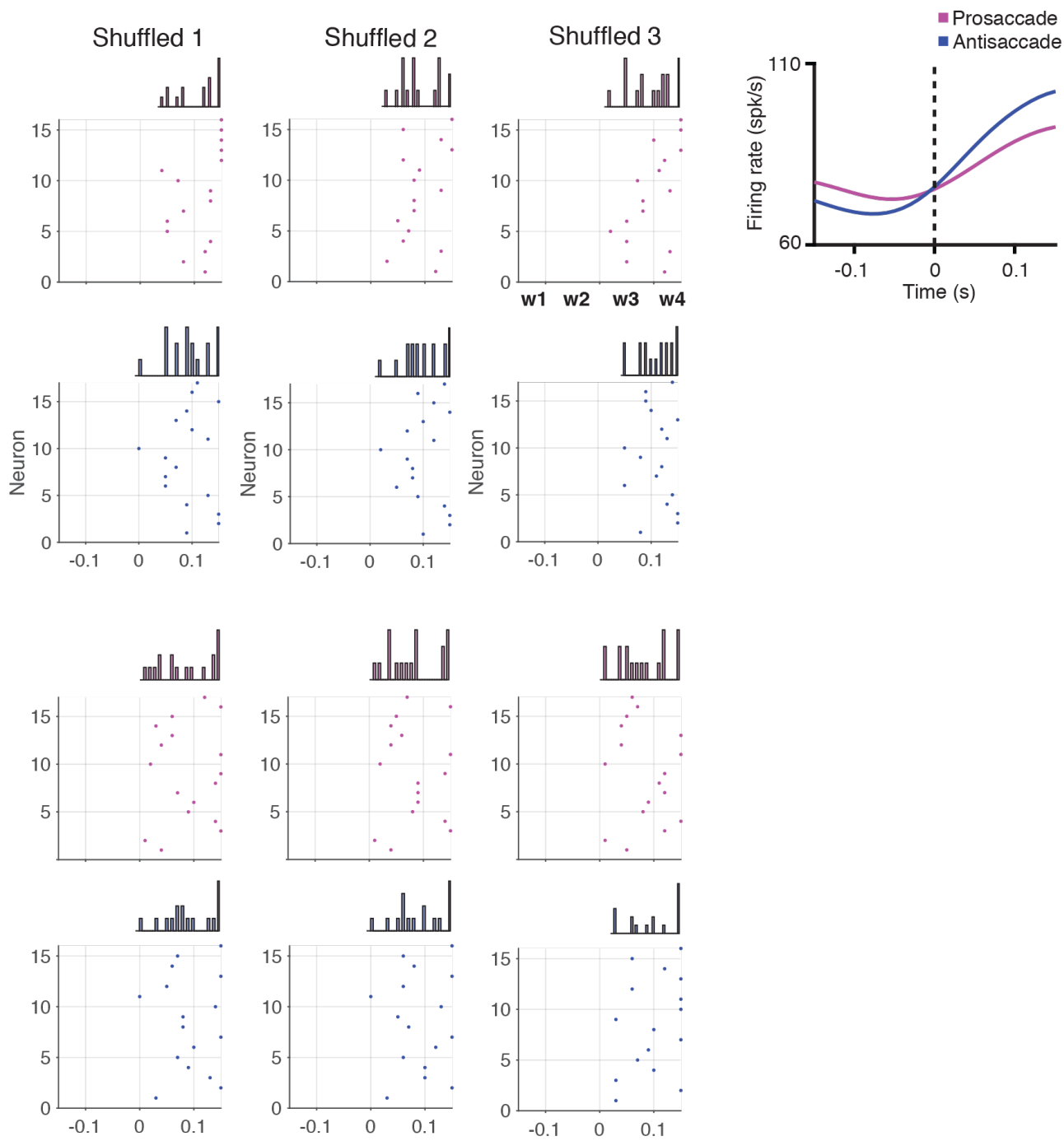

Supplementary Figure 4. PC maximum responses are stable across trials. a, Leftmost panel shows unsorted maximum response time for all medial cells including all trials. Rest of the panels were calculated by shuffling the order of the trials and selecting only $50 \%$ of them. b. Same as (a) for lateral cerebellum. c, One example cell from lateral cerebellum showing maximum responses at the end of the saccade window. 
Medial

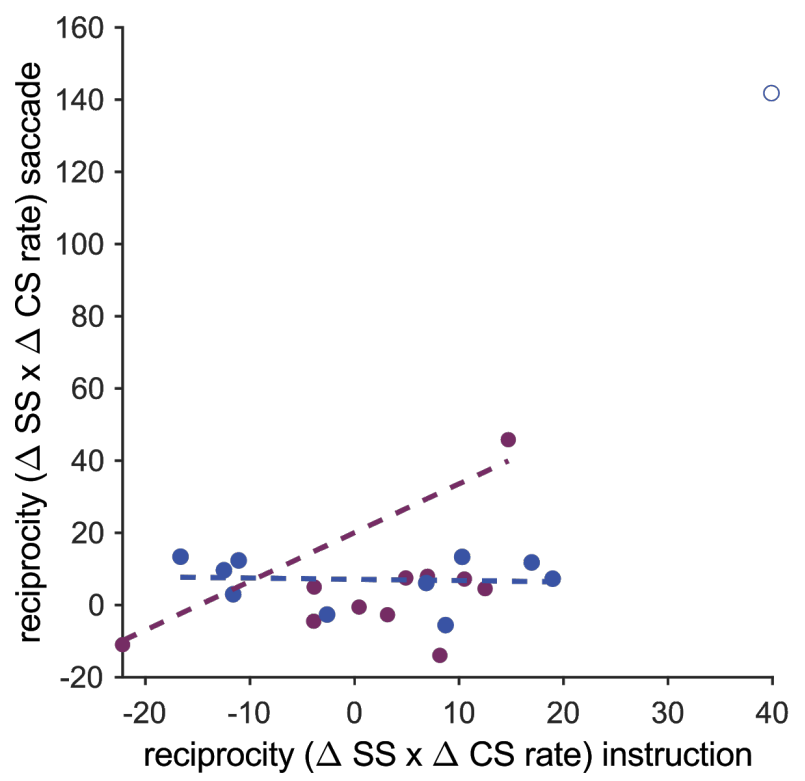

Lateral

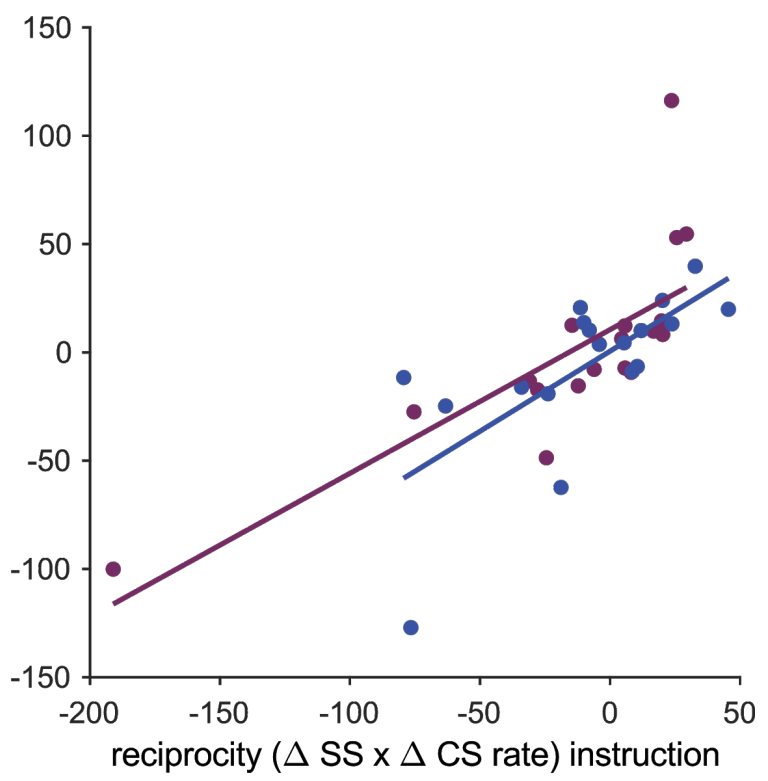

Supplementary Figure 5. Shared reciprocity between instruction period and saccade period.

To quantify the reciprocity between CS and SS responses the maximum change in CS firing rate in the windows of interest (instruction and saccade) were calculated. Subsequently, the associated maximum change in SS rate in a window of $300 \mathrm{~ms}$ around the peak CS rate was found. To quantify the reciprocity we calculated the product ( $\Delta$ CS rate $\mathrm{x} \Delta \mathrm{SS}$ rate). If there is a positive modulation of CS and negative modulation of SS and thus reciprocity this will give a negative value. If both CS and SS have positive modulation and thus no reciprocity this will give a positive value. Every dot represents one cell. One outlier was removed from the medial anti-saccade group, because it changed the coefficient of determination from $\mathrm{R}^{2}=0.44$ to $\mathrm{R}^{2}=0.01$ when removing the outlier, indicating that the whole association depended on that one value; for other coefficients of determination see Table below.

\begin{tabular}{|l|l|}
\hline & $\mathrm{R}^{2}$ \\
\hline Medial pro & 0.30 \\
\hline Medial anti & 0.01 \\
\hline Lateral pro & 0.59 \\
\hline Lateral anti & 0.48 \\
\hline
\end{tabular}




\section{Supplementary Tables}

\begin{tabular}{|c|c|c|c|c|c|}
\hline & & \multicolumn{2}{|c|}{ Medial } & \multicolumn{2}{|c|}{ Lateral } \\
\hline & & pro & anti & pro & anti \\
\hline \multirow{3}{*}{ Simple spikes } & Firing rate & $61.4( \pm 22.6)$ & $62.9( \pm 24.7)$ & $68( \pm 30)$ & $69.3( \pm 31.3)$ \\
\hline & \# trials & $\begin{array}{c}75( \pm 49) \\
6801\end{array}$ & $\begin{array}{c}71( \pm 47) \\
6431\end{array}$ & $\begin{array}{c}80( \pm 52) \\
5784\end{array}$ & $\begin{array}{c}76( \pm 50) \\
5471\end{array}$ \\
\hline & \# cells & \multicolumn{2}{|c|}{90} & \multicolumn{2}{|c|}{72} \\
\hline \multirow{3}{*}{$\begin{array}{l}\text { Complex } \\
\text { spikes }\end{array}$} & Firing rate & $1.08( \pm 0.55)$ & $1.11( \pm 0.48)$ & $0.91( \pm 0.23)$ & $0.92( \pm 0.22)$ \\
\hline & \# trials & $\begin{array}{c}192( \pm 187) \\
2114\end{array}$ & $\begin{array}{c}127( \pm 93) \\
1401\end{array}$ & $\begin{array}{c}120( \pm 38) \\
2343\end{array}$ & $\begin{array}{c}112( \pm 39) \\
2213\end{array}$ \\
\hline & \# cells & \multicolumn{2}{|c|}{11} & \multicolumn{2}{|c|}{18} \\
\hline
\end{tabular}

Supplementary Table 1. Summary of quantitative statistics of total population of neurons describing average firing rate of all neurons during the saccade period (Methods), number of trials per cell and grand total of trials, and number of cells per category.

Complex spike responses were only analyzed if the cell's CS firing rates was between 0.5 and $2.5 \mathrm{hz}$ over the entire recording and at least 5 correct trials were recorded per direction (8 pro direction and 8 anti directions). 


\begin{tabular}{|c|c|c|c|c|}
\hline & & \multicolumn{2}{|c|}{ Execution period } & \multirow{2}{*}{$\begin{array}{c}\text { Instruction period } \\
\text { Selected }\end{array}$} \\
\hline & & All & Selected & \\
\hline \multirow{2}{*}{$\begin{array}{l}\text { Medial } \\
(n=90)\end{array}$} & Facilitation & $\begin{array}{l}\text { Pro } 37(\sim 41 \%) \\
\text { Anti } 40(\sim 44 \%)\end{array}$ & $\begin{array}{l}\text { Pro } 16(\sim 18 \%) \\
\text { Anti } 17(\sim 19 \%)\end{array}$ & $\begin{array}{l}\text { Pro } 9(10 \%) \\
\text { Anti } 10(\sim 11 \%)\end{array}$ \\
\hline & Suppression & $\begin{array}{l}\text { Pro } 53(\sim 59 \%) \\
\text { Anti } 50(\sim 56 \%)\end{array}$ & $\begin{array}{l}\text { Pro } 18(\sim 20 \%) \\
\text { Anti } 10(\sim 11 \%)\end{array}$ & $\begin{array}{l}\text { Pro } 10(\sim 11 \%) \\
\text { Anti } 6(\sim 7 \%)\end{array}$ \\
\hline \multirow{2}{*}{$\begin{array}{l}\text { Lateral } \\
(n=72)\end{array}$} & Facilitation & $\begin{array}{l}\text { Pro } 37(\sim 51 \%) \\
\text { Anti } 34(\sim 48 \%)\end{array}$ & $\begin{array}{l}\text { Pro } 17(\sim 23 \%) \\
\text { Anti } 16(\sim 22 \%)\end{array}$ & $\begin{array}{l}\text { Pro } 12(\sim 17 \%) \\
\text { Anti } 13(\sim 18 \%)\end{array}$ \\
\hline & Suppression & $\begin{array}{l}\text { Pro } 35(\sim 49 \%) \\
\text { Anti } 38(\sim 52 \%)\end{array}$ & $\begin{array}{l}\text { Pro } 14(\sim 20 \%) \\
\text { Anti } 14(\sim 20 \%)\end{array}$ & $\begin{array}{l}\text { Pro } 10(\sim 14 \%) \\
\text { Anti } 11(\sim 15 \%)\end{array}$ \\
\hline
\end{tabular}

Supplementary Table 2. Quantification of SS activity profiles in medial and lateral cerebellum. PCs were classified into two groups based on whether their activity during execution (left columns) or instruction (right columns) increased (Facilitation) or decreased (Suppression) its neural activity (for definitions of time windows, see Methods). A cell could have a significant suppression activity for antisaccades but not for prosaccades. Columns indicated by "Selected" show the number of cells for which the modulation was significant, not the total number of cells. 


\begin{tabular}{|c|c|c|c|}
\hline & & $\begin{array}{c}\text { Medial cerebellum } \\
40 \text { cells }\end{array}$ & $\begin{array}{c}\text { Lateral cerebellum } \\
38 \mathrm{cells}\end{array}$ \\
\hline \multirow{5}{*}{$\infty \circlearrowleft$} & prosaccade trials only & $10(\sim 25 \%)$ & $9(\sim 24 \%)$ \\
\hline & antisaccade trials only & $6(\sim 15 \%)$ & $9(\sim 24 \%)$ \\
\hline & pro- and antisaccade & $9(\sim 23 \%)$ & $13(\sim 34 \%)$ \\
\hline & no modulation & $15(\sim 38 \%)$ & $7(\sim 18 \%)$ \\
\hline & total cells & $40(100 \%)$ & $38(100 \%)$ \\
\hline & & 11 cells & $18 \mathrm{cells}$ \\
\hline \multirow{5}{*}{$\cup \bullet$} & prosaccade trials only & $3(27 \%)$ & $4(\sim 22 \%)$ \\
\hline & antisaccade trials only & $3(\sim 18 \%)$ & $1(\sim 6 \%)$ \\
\hline & pro- and antisaccade & $2(\sim 18 \%)$ & $8(\sim 44 \%)$ \\
\hline & no directionality & $4(\sim 36 \%)$ & $5(\sim 28 \%)$ \\
\hline & total cells & $11(100 \%)$ & $18(100 \%)$ \\
\hline
\end{tabular}

Supplementary Table 3. Neuronal populations with directional preference for saccades split between SSs and CSs. A sinusoidal was fitted to the firing frequency in the saccade window. If the R2 of the fit was higher than 0.4 it was deemed significant. Inclusion criteria CS are the same as the previous table. Cells were only included if SS responses were on average over the whole between 15 and $170 \mathrm{hz}$ and at least 5 correct trials were available per direction ( 8 pro directions and 8 anti directions). 


\section{References}

1. Diamond, A. Executive functions. Annu. Rev. Psychol. 64, 135-168 (2013).

2. Munoz, D. P. \& Everling, S. Look away: the anti-saccade task and the voluntary control of eye movement. Nat. Rev. Neurosci. 5, 218-228 (2004).

3. Everling, S. \& Fischer, B. The antisaccade: a review of basic research and clinical studies. Neuropsychologia 36, 885-899 (1998).

4. Mitchell, J. P., Macrae, C. N. \& Gilchrist, I. D. Working memory and the suppression of reflexive saccades. J. Cogn. Neurosci. 14, 95-103 (2002).

5. Barton, J. J. S. et al. Antisaccades and task switching: studies of control processes in saccadic function in normal subjects and schizophrenic patients. Ann. N. Y. Acad. Sci. 956, 250-263 (2002).

6. Hakvoort Schwerdtfeger, R. M. et al. Preparatory neural networks are impaired in adults with attention-deficit/hyperactivity disorder during the antisaccade task. Neuroimage Clin $\mathbf{2}$, $63-78(2012)$.

7. Radant, A. D. et al. Antisaccade performance in schizophrenia patients, their first-degree biological relatives, and community comparison subjects: data from the COGS study. Psychophysiology 47, 846-856 (2010).

8. Barton, J. J. S., Pandita, M., Thakkar, K., Goff, D. C. \& Manoach, D. S. The relation between antisaccade errors, fixation stability and prosaccade errors in schizophrenia. Exp. Brain Res. 186, 273-282 (2008).

9. Bunge, S. A. et al. Neural circuitry underlying rule use in humans and nonhuman primates. J. Neurosci. 25, 10347-10350 (2005).

10. Everling, S. \& Johnston, K. Control of the superior colliculus by the lateral prefrontal 
cortex. Philos. Trans. R. Soc. Lond. B Biol. Sci. 368, 20130068 (2013).

11. Gottlieb, J. \& Goldberg, M. E. Activity of neurons in the lateral intraparietal area of the monkey during an antisaccade task. Nat. Neurosci. 2, 906-912 (1999).

12. Schlag-Rey, M., Amador, N., Sanchez, H. \& Schlag, J. Antisaccade performance predicted by neuronal activity in the supplementary eye field. Nature 390, 398-401 (1997).

13. Funahashi, S., Chafee, M. V. \& Goldman-Rakic, P. S. Prefrontal neuronal activity in rhesus monkeys performing a delayed anti-saccade task. Nature 365, 753-756 (1993).

14. Cutsuridis, V., Kumari, V. \& Ettinger, U. Antisaccade performance in schizophrenia: a neural model of decision making in the superior colliculus. Front. Neurosci. 8, 13 (2014).

15. Kelly, R. M. \& Strick, P. L. Cerebellar loops with motor cortex and prefrontal cortex of a nonhuman primate. J. Neurosci. 23, 8432-8444 (2003).

16. Peterburs, J. et al. Cerebellar lesions alter performance monitoring on the antisaccade task-an event-related potentials study. Neuropsychologia 50, 379-389 (2012).

17. Kunimatsu, J., Suzuki, T. W. \& Tanaka, M. Implications of Lateral Cerebellum in Proactive Control of Saccades. Journal of Neuroscience 36, 7066-7074 (2016).

18. Brunamonti, E. et al. Cerebellar damage impairs executive control and monitoring of movement generation. PLoS One 9, e85997 (2014).

19. Tanaka, H., Harada, M., Arai, M. \& Hirata, K. Cognitive dysfunction in cortical cerebellar atrophy correlates with impairment of the inhibitory system. Neuropsychobiology 47, 206$211(2003)$.

20. Dacre, J. et al. Cerebellar-recipient motor thalamus drives behavioral context-specific movement initiation. bioRxiv 802124 (2019) doi:10.1101/802124.

21. Gao, Z. et al. A cortico-cerebellar loop for motor planning. Nature 563, 113-116 (2018). 
22. Giovannucci, A. et al. Cerebellar granule cells acquire a widespread predictive feedback signal during motor learning. Nat. Neurosci. 20, 727-734 (2017).

23. Kostadinov, D., Beau, M., Pozo, M. B. \& Häusser, M. Predictive and reactive reward signals conveyed by climbing fiber inputs to cerebellar Purkinje cells. Nat. Neurosci. 22, 950-962 (2019).

24. Deverett, B., Koay, S. A., Oostland, M. \& Wang, S. S.-H. Cerebellar involvement in an evidence-accumulation decision-making task. Elife 7, (2018).

25. Ashmore, R. C. \& Sommer, M. A. Delay activity of saccade-related neurons in the caudal dentate nucleus of the macaque cerebellum. J. Neurophysiol. 109, 2129-2144 (2013).

26. Gao, Z. et al. Response to 'Fallacies of Mice Experiments'. Neuroinformatics vol. 17 475478 (2019).

27. Miall, R. C., Weir, D. J., Wolpert, D. M. \& Stein, J. F. Is the cerebellum a smith predictor? J. Mot. Behav. 25, 203-216 (1993).

28. Thach, W. T. On the mechanism of cerebellar contributions to cognition. Cerebellum $\mathbf{6}$, 163-167 (2007).

29. Ito, M. Control of mental activities by internal models in the cerebellum. Nat. Rev. Neurosci. 9, 304-313 (2008).

30. Chabrol, F. P., Blot, A. \& Mrsic-Flogel, T. D. Cerebellar Contribution to Preparatory Activity in Motor Neocortex. Neuron 103, 506-519.e4 (2019).

31. De Schutter, E. Fallacies of Mice Experiments. Neuroinformatics 17, 181-183 (2019).

32. Strick, P. L., Dum, R. P. \& Fiez, J. A. Cerebellum and nonmotor function. Annu. Rev. Neurosci. 32, 413-434 (2009).

33. Caligiore, D. et al. Consensus Paper: Towards a Systems-Level View of Cerebellar 
Function: the Interplay Between Cerebellum, Basal Ganglia, and Cortex. Cerebellum 16, 203-229 (2017).

34. Sendhilnathan, N., Semework, M., Goldberg, M. E. \& Ipata, A. E. Neural Correlates of Reinforcement Learning in Mid-lateral Cerebellum. Neuron 106, 188-198.e5 (2020).

35. De Zeeuw, C. I. Bidirectional learning in upbound and downbound microzones of the cerebellum. Nat. Rev. Neurosci. 22, 92-110 (2021).

36. Voogd, J., Schraa-Tam, C. K., van der Geest, J. N. \& De Zeeuw, C. I. Visuomotor cerebellum in human and nonhuman primates. Cerebellum 11, 392-410 (2012).

37. Heiney, S. A., Kim, J., Augustine, G. J. \& Medina, J. F. Precise control of movement kinematics by optogenetic inhibition of Purkinje cell activity. J. Neurosci. 34, 2321-2330 (2014).

38. ten Brinke, M. M. et al. Evolving Models of Pavlovian Conditioning: Cerebellar Cortical Dynamics in Awake Behaving Mice. Cell Rep. 13, 1977-1988 (2015).

39. Yang, Y. \& Lisberger, S. G. Purkinje-cell plasticity and cerebellar motor learning are graded by complex-spike duration. Nature 510, 529-532 (2014).

40. Ten Brinke, M. M. et al. Dynamic modulation of activity in cerebellar nuclei neurons during pavlovian eyeblink conditioning in mice. Elife 6, (2017).

41. Herzfeld, D. J., Kojima, Y., Soetedjo, R. \& Shadmehr, R. Encoding of action by the Purkinje cells of the cerebellum. Nature 526, 439-442 (2015).

42. Herzfeld, D. J., Kojima, Y., Soetedjo, R. \& Shadmehr, R. Encoding of error and learning to correct that error by the Purkinje cells of the cerebellum. Nat. Neurosci. 21, 736-743 (2018).

43. Harvey, C. D., Coen, P. \& Tank, D. W. Choice-specific sequences in parietal cortex during 
a virtual-navigation decision task. Nature 484, 62-68 (2012).

44. Ikegaya, Y. et al. Synfire chains and cortical songs: temporal modules of cortical activity. Science 304, 559-564 (2004).

45. Jin, D. Z., Fujii, N. \& Graybiel, A. M. Neural representation of time in cortico-basal ganglia circuits. Proc. Natl. Acad. Sci. U. S. A. 106, 19156-19161 (2009).

46. Amador, N., Schlag-Rey, M. \& Schlag, J. Primate antisaccades. I. Behavioral characteristics. J. Neurophysiol. 80, 1775-1786 (1998).

47. Goldring, J. \& Fischer, B. Reaction times of vertical prosaccades and antisaccades in gap and overlap tasks. Exp. Brain Res. 113, 88-103 (1997).

48. Coe, B. C. \& Munoz, D. P. Mechanisms of saccade suppression revealed in the anti-saccade task. Philos. Trans. R. Soc. Lond. B Biol. Sci. 372, (2017).

49. Badura, A. et al. Climbing fiber input shapes reciprocity of Purkinje cell firing. Neuron 78, 700-713 (2013).

50. Sun, Z., Smilgin, A., Junker, M., Dicke, P. W. \& Thier, P. The same oculomotor vermal Purkinje cells encode the different kinematics of saccades and of smooth pursuit eye movements. Sci. Rep. 7, 40613 (2017).

51. Soetedjo, R., Kojima, Y. \& Fuchs, A. F. How cerebellar motor learning keeps saccades accurate. J. Neurophysiol. 121, 2153-2162 (2019).

52. De Zeeuw, C. I. \& Ten Brinke, M. M. Motor Learning and the Cerebellum. Cold Spring Harb. Perspect. Biol. 7, (2015).

53. Mokler, A. \& Fischer, B. The recognition and correction of involuntary prosaccades in an antisaccade task. Exp. Brain Res. 125, 511-516 (1999).

54. Ron, S. \& Robinson, D. A. Eye movements evoked by cerebellar stimulation in the alert 
monkey. J. Neurophysiol. 36, 1004-1022 (1973).

55. Piu, P. et al. The cerebellum improves the precision of antisaccades by a latency-duration trade-off. Prog. Brain Res. 249, 125-139 (2019).

56. Thier, P., Dicke, P. W., Haas, R., Thielert, C.-D. \& Catz, N. The role of the oculomotor vermis in the control of saccadic eye movements. Ann. N. Y. Acad. Sci. 978, 50-62 (2002).

57. Kornhuber, H. H. Motor functions of cerebellum and basal ganglia: the cerebellocortical saccadic (ballistic) clock, the cerebellonuclear hold regulator, and the basal ganglia ramp (voluntary speed smooth movement) generator. Kybernetik 8, 157-162 (1971).

58. Everling, S., Dorris, M. C. \& Munoz, D. P. Neuronal Activity in Monkey Superior Colliculus during an Antisaccade Task. in Current Oculomotor Research: Physiological and Psychological Aspects (eds. Becker, W., Deubel, H. \& Mergner, T.) 17-23 (Springer US, 1999).

59. Marple-Horvat, D. E. \& Stein, J. F. Neuronal activity in the lateral cerebellum of trained monkeys, related to visual stimuli or to eye movements. J. Physiol. 428, 595-614 (1990).

60. Gao, Z., van Beugen, B. J. \& De Zeeuw, C. I. Distributed synergistic plasticity and cerebellar learning. Nat. Rev. Neurosci. 13, 619-635 (2012).

61. McElligott, J. G. \& Keller, E. L. Cerebellar vermis involvement in monkey saccadic eye movements: microstimulation. Exp. Neurol. 86, 543-558 (1984).

62. Noda, H. \& Fujikado, T. Involvement of Purkinje cells in evoking saccadic eye movements by microstimulation of the posterior cerebellar vermis of monkeys. J. Neurophysiol. 57, 1247-1261 (1987).

63. Thier, P. \& Möck, M. The oculomotor role of the pontine nuclei and the nucleus reticularis tegmenti pontis. Prog. Brain Res. 151, 293-320 (2006). 
64. Ohki, M. et al. Role of primate cerebellar hemisphere in voluntary eye movement control revealed by lesion effects. J. Neurophysiol. 101, 934-947 (2009).

65. Catz, N., Dicke, P. W. \& Thier, P. Cerebellar-dependent motor learning is based on pruning a Purkinje cell population response. Proc. Natl. Acad. Sci. U. S. A. 105, 7309-7314 (2008).

66. Cotti, J. et al. Adaptation of reactive and voluntary saccades: different patterns of adaptation revealed in the antisaccade task. J. Physiol. 587, 127-138 (2009).

67. Lévy-Bencheton, D., Pisella, L., Salemme, R., Tilikete, C. \& Pélisson, D. Plastic modification of anti-saccades: adaptation of saccadic eye movements aimed at a virtual target. J. Neurosci. 33, 13489-13497 (2013).

68. Welsh, J. P., Lang, E. J., Suglhara, I. \& Llinas, R. Dynamic organization of motor control within the olivocerebellar system. Nature 374, 453-457 (1995).

69. Van Der Giessen, R. S. et al. Role of olivary electrical coupling in cerebellar motor learning. Neuron 58, 599-612 (2008).

70. Wang, X., Yu, S.-Y., Ren, Z., De Zeeuw, C. I. \& Gao, Z. A FN-MdV pathway and its role in cerebellar multimodular control of sensorimotor behavior. Nat. Commun. 11, 6050 (2020)

71. Kunimatsu, J., Suzuki, T. W., Ohmae, S. \& Tanaka, M. Different contributions of preparatory activity in the basal ganglia and cerebellum for self-timing. Elife 7, (2018).

72. Saleem, K. S. \& Logothetis, N. K. A Combined MRI and Histology Atlas of the Rhesus Monkey Brain in Stereotaxic Coordinates. (Academic Press, 2012).

73. De Zeeuw, C. I. et al. Spatiotemporal firing patterns in the cerebellum. Nat. Rev. Neurosci. 12, 327-344 (2011).

74. Nyström, M. \& Holmqvist, K. An adaptive algorithm for fixation, saccade, and glissade 
detection in eyetracking data. Behav. Res. Methods 42, 188-204 (2010).

75. Silverman, B. W. Density Estimation for Statistics and Data Analysis. (1986)

doi:10.1007/978-1-4899-3324-9.

76. Dash, S., Dicke, P. W. \& Thier, P. A vermal Purkinje cell simple spike population response encodes the changes in eye movement kinematics due to smooth pursuit adaptation. Front.

Syst. Neurosci. 7, 3 (2013). 